Please do not remove this page

RMIT

UNIVERSITY

\title{
Transformations for nonideal uniform circular arrays operating in correlated signal environments
}

Lau, Buon Kiong; Leung, Yee Hong; Liu, Yanqun; Teo, Kok Lay

https://researchrepository.rmit.edu.au/esploro/outputs/9921859748901341/filesAndLinks?institution=61RMIT_INST\&index=null

Lau, B. K., Leung, Y. H., Liu, Y., \& Teo, K. L. (2006). Transformations for nonideal uniform circular arrays operating in correlated signal environments. IEEE Transactions on Signal Processing, 54(1), 34-48.

https://doi.org/10.1109/tsp.2005.861104

Published Version: https://doi.org/10.1109/tsp.2005.861104

Repository homepage: https://researchrepository.rmit.edu.au

(c) 2006 IEEE. Personal use of this material is permitted. However, permission to reprint/republish this material for advertising or promotional purposes or for creating new collective works for resale or redistribution to servers or lists, or to reuse any copyrighted component of this work in other works must be obtained from the IEEE.

Downloaded On 2023/04/26 23:02:46 +1000 


\title{
Transformations for Nonideal Uniform Circular Arrays Operating in Correlated Signal Environments
}

\author{
Buon Kiong Lau, Member, IEEE, Yee Hong Leung, Member, IEEE, Yanqun Liu, and \\ Kok Lay Teo, Senior Member, IEEE
}

\begin{abstract}
The Davies transformation is a method to transform the steering vector of a uniform circular array (UCA) to one with Vandermonde form. As such, it allows techniques such as spatial smoothing for direction-of-arrival (DOA) estimation in a correlated signal environment, developed originally for uniform linear arrays, to be applied to UCAs. However, the Davies transformation can be highly sensitive to perturbations of the underlying array model. This paper presents a method for deriving a more robust transformation using optimization techniques. The effectiveness of the method is illustrated through a number of DOA estimation examples.
\end{abstract}

Index Terms-Correlated signals, direction-of-arrival (DOA) estimation, quadratic semi-infinite programming, robustness, uniform circular arrays (UCAs).

\section{INTRODUCTION}

B Y virtue of their geometry, uniform circular arrays (UCAs) are able to provide $360^{\circ}$ of coverage in the azimuth plane. Moreover, they are known to be isotropic. That is, they can estimate the direction of arrival (DOA) of an incident signal with uniform resolution in the azimuth plane [1]. As such, UCAs are eminently suitable for applications such as radar, sonar, and wireless communications [2]. This advantage of the UCAs is counterbalanced, however, by the unaccommodating mathematical structure of their steering vectors. In particular, many important array signal processing techniques that have been developed, such as Dolph-Chebyshev beampattern design [3], and spatial smoothing for DOA estimation and adaptive and optimum beamforming in a correlated signal environment [4]-[6], apply only to uniform linear arrays (ULAs) whose

Manuscript received November 14, 2003; revised November 15, 2004. This work was supported in part by the Australian Telecommunications Cooperative Research Centre (ATcrc), the Australian Research Council under Grant A00105530, and the Hong Kong Research Grants Council under Grant PolyU $5101 / 02 \mathrm{E}$. The associate editor coordinating the review of this manuscript and approving it for publication was Dr. Constantinos B. Papadias.

B. K. Lau is with the Department of Electroscience, Lund University, SE-221 00 Lund, Sweden (e-mail: bkl@es.lth.se).

Y. H. Leung is with the Department of Electrical and Computer Engineering, Curtin University of Technology, Bentley WA 6102, Australia (e-mail: leungyh@ece.curtin.edu.au).

Y. Liu is with the Department of Mathematics and Statistics, RMIT University, Melbourne, VIC 3001, Australia (e-mail: yanqun.liu@ rmit.edu.au).

K. L. Teo was with the Department of Applied Mathematics, Hong Kong Polytechnic University, Hung Hom, Kowloon, Hong Kong, China. He is now with the Department of Mathematics and Statistics, Curtin University of Technology, Bentley WA 6102, Australia (e-mail: K.L.Teo@curtin.edu.au).

Digital Object Identifier 10.1109/TSP.2005.861104 steering vector has a Vandermonde structure. The steering vector of a UCA is not Vandermonde.

Currently, there are two popular approaches to transform the steering vector of a UCA to Vandermonde form. The interpolated array approach, first proposed by Bronez [7], and later, under different formulations, by Friedlander [8], Pesavento et al. [9], and Cook et al. [10]-[12], involves fitting the steering vector of the UCA to that of a ULA ${ }^{1}$ over an angular sector in the azimuth. Thus, it involves sector-by-sector processing, which can be inconvenient.

The second approach, proposed by Davies [13], involves transforming the steering vector of the UCA to that of a virtual array, which we termed the Davies array. The key points about this array are as follows:

1) it covers the entire azimuth plane and so does not require sector-by-sector processing;

2) its steering vector is Vandermonde, or approximately so;

3) unlike the virtual ULA of the interpolated array approach, it has no physical interpretation, i.e., it is a purely mathematical construct and is said to exist in mode space [2].

In [14], we use the Davies transformation to design DolphChebyshev beampatterns for UCAs, while in [2], [15] and [16], it is used to enable DOA estimation and optimum beamforming for UCAs in a correlated signal environment.

Apart from the interpolated array and Davies array, Bührens et al. [17] recently proposed another virtual array which, like the Davies array, has no physical interpretation. The steering vector of Bührens' array is also not Vandermonde though it is shift invariant to enable application of the ESPRIT DOA estimation algorithm. The thrust of Bührens' work is to reduce the bias in the DOA estimates that results from Friedlander's interpolated array. However, as with the interpolated array, Bührens' array covers only a finite sector in the azimuth and hence also requires sector-by-sector processing.

Returning to the Davies array, we remark here that in [13], it was assumed tacitly that the antenna elements all have the same omnidirectional response, the electronics associated with each antenna element are identical, the antenna elements are located at their correct positions, and there is no mutual coupling between the antenna elements. Clearly, in any real implementation, none of these assumptions will hold. In [18], it is shown that when small errors are introduced into the model of an ideal UCA, as represented by a perturbation of its steering vector,

\footnotetext{
${ }^{1}$ Although the steering vector of the UCA can be fitted to other array geometries, the interest here is on arrays with Vandermonde steering vectors.
} 
the performance of the virtual array can degrade appreciably. In [2], it is pointed out that these real-world effects can be ameliorated somewhat by calibration, but calibration is never perfect because of noise and systematic errors in the calibration data. In addition, changes in the environment can take a "perfectly" calibrated array out of calibration. In this paper, we show that the "calibrated Davies" transformation is also not robust.

Indeed, the whole issue of lack of robustness can be related to the phenomenon of "resonance" in classical wave mechanics [19]. Resonance is an inherent limitation of array processing and will arise for certain array geometries, that is, the placement of the antenna elements relative to the operating wavelength of the array. In the case of UCAs, these correspond to the number of virtual array elements, the number of elements in the actual array, and the array's normalized radius (normalized with respect to the operating wavelength of the array). While in [2] and [20] it was pointed out that resonance can be circumvented with a careful choice of array geometries, or by using directional array elements, practical considerations such as hardware cost and the size of the platform where the array is to be mounted [8] may restrict the application of these methods. Moreover, it may not even be possible to avoid resonance altogether in applications involving wide-band signals or two-dimensional (2-D) (azimuth-elevation) operations since these applications involve a range of normalized radii due to the range of signal frequencies or the projection of the UCA antenna element locations to different elevation planes.

It is worthwhile pointing out here that, mathematically, the output signals of the antenna array can also be modeled as being the result of applying a sampling matrix to a representation of the propagating signal wavefield [19]. Resonance manifests itself as a loss of rank in the sampling matrix thus lowering the number of signals the UCA can resolve. However, this loss of rank does not necessarily imply a degradation in the performance of DOA estimation algorithms if the algorithms are applied to the array output directly. Therefore, it seems the idea of transforming the array output is rather pointless. Our argument is that to perform DOA estimation from the array output directly in a highly correlated signal environment, it is necessary to employ computationally intensive techniques such as the maximum-likelihood methods [21]. If the aim is to use simple DOA estimation methods such as MUSIC with spatial smoothing, then the transformation procedure is unavoidable.

The aim of this paper is to find, through global optimization techniques [22], [23], an alternative transformation that is more robust. The basis of our approach is to sacrifice the Vandermonde approximation error of the virtual array steering vector for improved robustness. The robustness of the new transformation is demonstrated by performing DOA estimation with spatial smoothing and MUSIC in a correlated signal environment. Moreover, the statistical performances of our proposal are also evaluated against root-weighted subspace fitting (WSF) (or MODE) [24].

This paper is organized as follows. In Section II, we present the optimization problem which we formulated to obtain the robust Davies transformation matrix and discuss a number of issues that determines the robustness of the Davies transformation. The solution of the optimization problem, using a quadratic semi-infinite programming technique, is described in an Appendix. The robust transformation matrix has a number of symmetry properties that can be exploited to reduce greatly the computational complexity of the numerical optimization problem. We state these properties in Section III. In Section IV, we demonstrate the effectiveness of our robustness approach through a number of numerical examples and show the calibration procedure described in [2] is also nonrobust. Finally, Section $\mathrm{V}$ concludes the paper.

Before proceeding further, we wish to emphasize here that both the Davies transformation matrix and our proposed transformation matrix are fixed matrices. They are determined offline during the design stage and depend only on the ideal or nominal array geometry and the number of elements in the virtual array. They are not updated during the operation of the antenna array.

\section{PROBLEM FORMULATION}

\section{A. The Davies Transformation}

Consider a UCA with $N$ elements and radius $r$ located in the $x y$ or azimuth plane. Suppose the center of the array coincides with the origin of the spatial coordinate system, and azimuth angles are measured anti-clockwise from the $x$ axis. Suppose element 1 of the array is placed on the $x$ axis, i.e., at $\theta=0^{\circ}$. Denote by $\mathbf{a}(\theta)$ the array response (or steering) vector of the UCA in response to a narrow-band signal of wavelength $\lambda$ arriving from angle $\theta \in[-\pi, \pi)$ in the azimuth plane. The $n$th component of $\mathbf{a}(\theta)$ is given by

$$
[\mathbf{a}(\theta)]_{n}=G_{n}(\theta) \exp \left\{j k r \cos \left(\theta-\gamma_{n}\right)\right\}
$$

where $G_{n}(\theta)$ is the complex gain pattern of the $n$th element, $k=2 \pi / \lambda$ is the wavenumber, $\gamma_{n}$ is the angular position of the $n$th array element

$$
\gamma_{n}=\frac{2 \pi(n-1)}{N}
$$

and to circumvent spatial aliasing, it is necessary that the interelement spacing ${ }^{2}$ is less than $\lambda / 2$ [15], [19], or alternatively, $N>4 \pi r / \lambda$.

Suppose the array elements are identical and omnidirectional such that $G_{n}(\theta)=1, n=1, \ldots, N$. In [13], Davies proposed transforming the antenna element outputs as shown in Fig. 1, where $x_{1}, \ldots, x_{N}$ represent the baseband complex output signals of the "real" or physical array, and $y_{1}, \ldots, y_{M}, M<N$ represent the baseband complex output signals of the virtual array. In [2], it is shown that if the transformation matrix $\mathbf{T}$ is given by

$$
\mathbf{T}=\mathbf{T}_{\mathrm{D}} \triangleq \mathbf{J F}
$$

where $\mathbf{J} \in \mathbb{C}^{M \times M}$ and $\mathbf{F} \in \mathbb{C}^{M \times N}$ are given by

$$
\begin{aligned}
\mathbf{J} & =\operatorname{diag}\left\{\left[j^{m-1-M_{o}} \sqrt{N} J_{m-1-M_{o}}(k r)\right]^{-1}\right\} \\
{[\mathbf{F}]_{\mathrm{mn}} } & =\frac{1}{\sqrt{N}} e^{j 2 \pi\left(m-1-M_{o}\right)(n-1) / N}
\end{aligned}
$$

${ }^{2}$ For a UCA, the inter-element spacing $d=2 r \sin (\pi / N)$. 


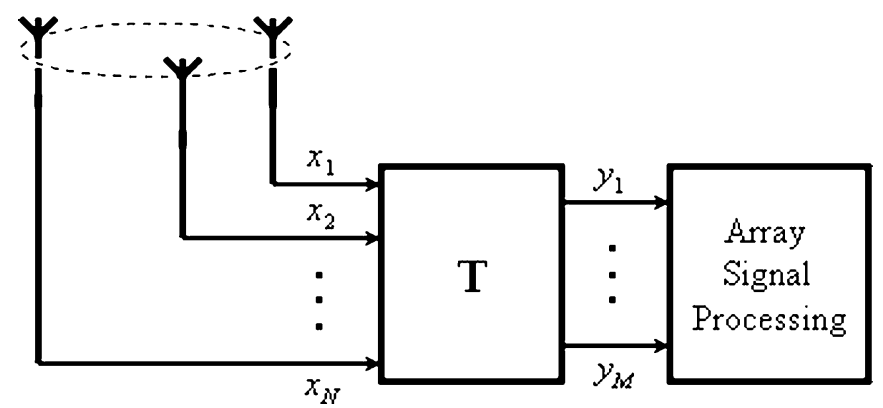

Fig. 1. Transformation for UCAs.

and where $m=1, \ldots, M, n=1, \ldots, N J_{p}(\cdot)$ denotes a $p$ th-order Bessel function of the first kind, and

$$
M_{o}=\frac{M-1}{2} \in \mathbb{Z}
$$

then the $M$-dimensional steering vector of the virtual or Davies array, which we denote by $\mathbf{b}_{\mathrm{D}}(\theta)$, will take on, approximately, the Vandermonde form

$$
\begin{aligned}
\mathbf{b}_{\mathrm{D}}(\theta) & =\mathbf{T}_{\mathrm{D}} \mathbf{a}(\theta) \\
& \cong\left[\begin{array}{lllllll}
e^{-j M_{o} \theta} & \cdots & e^{-j \theta} & 1 & e^{j \theta} & \cdots & e^{+j M_{o} \theta}
\end{array}\right]^{T} .
\end{aligned}
$$

Note, in view of (6), $M$ is odd. An appropriate choice for $M_{o}$, and hence $M$ by (6), for some predetermined $\delta$, is given by [2]

$$
\max \left\{M_{o} \| M_{o} \leq \frac{N-1}{2} \text { and } \frac{\left|J_{M_{o}-N}(k r)\right|}{\left|J_{M_{o}}(k r)\right|}<\delta\right\}
$$

where the first inequality relates to the spatial sampling condition [15], [19], and the second inequality determines the accuracy of the approximation in (7).

To facilitate our subsequent discussions, we define the vector

$$
\mathbf{b}(\theta) \triangleq\left[\begin{array}{lllllll}
e^{-j M_{o} \theta} & \cdots & e^{-j \theta} & 1 & e^{j \theta} & \cdots & e^{+j M_{o} \theta}
\end{array}\right]^{T} .
$$

\section{B. Problem Statement}

The lack of robustness of the Davies transformation can be traced to the construction of $\mathbf{J}$. As can be seen from (4), for certain choices of $m, M_{o}$, and $k r$, the magnitude of one or more of the diagonal elements of $\mathbf{J}$ can approach infinity as the corresponding value of $J_{m-1-M_{o}}(k r)$ approaches zero. Accordingly, the norm of $\mathbf{T}_{\mathrm{D}}$ can become very large. However, the square of the norm of $\mathbf{T}_{\mathrm{D}}$ gives a measure of the noise amplification of the transformation matrix. Therefore, for a $\mathbf{T}_{\mathrm{D}}$ with large norm, small perturbations in $\mathbf{a}(\theta)$ will translate to large perturbations in $\mathbf{b}_{\mathrm{D}}(\theta)$.

Based on the above observation, we formulate the following quadratic semi-infinite optimization problem to find a more robust transformation matrix. The basic idea is to trade off the approximation error in the transformation of $\mathbf{a}(\theta)$ for robustness.

Denote the robust transformation matrix by $\mathbf{T}_{\mathrm{R}} \in \mathbb{C}^{M \times N}$. We find $\mathbf{T}_{\mathrm{R}}$ as follows:

\section{Problem $(\mathcal{P} 1)$ :}

$$
\min _{\mathbf{T}_{\mathrm{R}}}\left\|\mathbf{T}_{\mathrm{R}}\right\|_{F}^{2}
$$

subject to

$$
\left|\mathbf{T}_{\mathrm{R}} \mathbf{a}(\theta)-\mathbf{b}(\theta)\right|_{\mathbb{C}} \leq \varepsilon, \quad \forall \theta \in[-\pi, \pi]
$$

where $\varepsilon=\left[\begin{array}{llll}\varepsilon_{1} & \varepsilon_{2} & \cdots & \varepsilon_{M}\end{array}\right]^{T}, \varepsilon_{m} \in \mathbb{R}_{+}, m=1, \ldots, M$, $\|\cdot\|_{F}$ denotes the Frobenius norm, and $|\cdot|_{\mathbb{C}}$ denotes the vector of complex absolute value norms defined by

$$
\begin{aligned}
&|\mathbf{x}|_{\mathbb{C}}^{T}=\left[\operatorname { m a x } \left\{\left|\operatorname{Re}\left\{x_{1}\right\}\right|,\right.\right.\left.\left|\operatorname{Im}\left\{x_{1}\right\}\right|\right\} \cdots \\
&\left.\max \left\{\left|\operatorname{Re}\left\{x_{M}\right\}\right|,\left|\operatorname{Im}\left\{x_{M}\right\}\right|\right\}\right]
\end{aligned}
$$

where $\mathbf{x} \in \mathbb{C}^{M}$ and $x_{m}$ is the $m$ th element of $\mathbf{x}$.

The salient feature of the above formulation is that the rows of $\mathbf{T}_{\mathrm{R}}$ are not coupled. Accordingly, $(\mathcal{P} 1)$ can be solved efficiently, row by row, as follows:

Problem $(\mathcal{P} 2)$ : For $m=1, \ldots, M$,

$$
\min _{\mathbf{t}_{m}}\left\|\mathbf{t}_{m}\right\|_{F}^{2}
$$

subject to

$$
\begin{aligned}
& \left|\operatorname{Re}\left\{e_{m}(\theta)\right\}\right| \leq \varepsilon_{m} \quad \text { and } \\
& \left|\operatorname{Im}\left\{e_{m}(\theta)\right\}\right| \leq \varepsilon_{m}, \quad \forall \theta \in[-\pi, \pi]
\end{aligned}
$$

where

$$
\begin{aligned}
e_{m}(\theta) & =\mathbf{t}_{m}^{T} \mathbf{a}(\theta)-b_{m}(\theta) \\
b_{m}(\theta) & =[\mathbf{b}(\theta)]_{m}
\end{aligned}
$$

and $\mathbf{t}_{m}^{T}$ is the $m$ th row of $\mathbf{T}_{\mathrm{R}}$.

Problem $(\mathcal{P} 2)$ results in $M$ quadratic semi-infinite programming problems. In Appendix I, we summarize the dual parametrization method [22], [23] to solve these problems. This method has significant advantages over other existing semi-infinite programming methods [25].

\section{Remarks:}

1) If for a given $m$, the feasible set of the corresponding subproblem of $(\mathcal{P} 2)$ is nonempty, then that subproblem has a unique solution. This follows since the cost function of the subproblem is strictly convex and the constraints define a convex region in $\mathbf{t}_{m}$.

2) If for a given $m, \varepsilon_{m} \geq 1$, then for that $m,(\mathcal{P} 2)$ has the trivial solution $\mathbf{t}_{m}=\mathbf{0}$ since $\left|b_{m}(\theta)\right|=1$.

\section{Design for Robustness}

The robustness of $\mathbf{T}_{R}$ depends on its norm. As a rough guide, the square of the norm of each row of $\mathbf{T}_{\mathrm{R}}$ should not greatly exceed $N / M$. The reasoning is as follows. Suppose array imperfection can be modeled as a (complex) noise output from the antenna elements, and the real and imaginary parts of these "noise" terms are independent with identical variance $\sigma_{x}^{2}$ and the "noise" terms from the antenna elements are mutually independent. The total "noise" from the $N$-element array is then given by $2 N \sigma_{x}^{2}$. Suppose the robust transformation matrix has Frobenius norm $\left\|\mathbf{T}_{\mathrm{R}}\right\|_{F}$. The total "noise" at the output of the virtual array is then given by $2\left\|\mathbf{T}_{\mathrm{R}}\right\|_{F}^{2} \sigma_{x}^{2}$. If the transformation is required to not increase "noise," then we require $2 N \sigma_{x}^{2} \geq 2\left\|\mathbf{T}_{\mathrm{R}}\right\|_{F}^{2} \sigma_{x}^{2}$, or $\left\|\mathbf{T}_{\mathrm{R}}\right\|_{F}^{2} \leq N$. Finally, suppose the "noise gain" is distributed 
uniformly over the elements of the virtual array. This yields $\left\|\mathbf{t}_{m}\right\|_{F}^{2}=\left\|\mathbf{T}_{\mathrm{R}}\right\|_{F}^{2} / M \leq N / M$.

The above consideration leads to the following strategy to make the Davies transformation matrix $\mathbf{T}_{\mathrm{D}}$ robust. We first compute $\mathbf{T}_{\mathrm{D}}$ and then check its row norms. We retain as many rows as possible since $\mathbf{T}_{\mathrm{D}}$ gives the best $L^{2}$ approximation of the UCA steering vector in mode space [19]. We replace only those rows whose norm is excessively large with rows found by solving the corresponding subproblems of $(\mathcal{P} 2)$. Suppose row $m$ of $\mathbf{T}_{\mathrm{D}}$ is to be replaced. We first set the optimization parameter $\varepsilon_{m}$ to 0.7 . We then either increase or decrease $\varepsilon_{m}$ depending on the norm of $\mathbf{t}_{m}$ found by solving $(\mathcal{P} 2)$ and the performance of the subsequent DOA estimation procedure.

\section{Some General Remarks}

1) To accomplish DOA estimation in both azimuth and elevation, a separate $\mathbf{T}_{\mathrm{R}}$ (or $\mathbf{T}_{\mathrm{D}}$ ) will be required for each elevation angle [15].

2) Calibration data can be used to find a calibration matrix $\mathbf{C}$ (see, for example, [2], [26], and [27]). The transformation matrix is then given by $\mathbf{T}=\mathbf{T}_{\mathrm{D}} \mathbf{C}$ or $\mathbf{T}=\mathbf{T}_{\mathrm{R}} \mathbf{C}$. This step can be performed, however, only if $\mathbf{C}$ is well conditioned, as otherwise it will give rise to large norms in the rows, or some of the rows, of $\mathbf{T}$.

3) Alternatively, similar to [2], calibration data can be incorporated into the robust matrix $\mathbf{T}_{\mathrm{R}}$ by replacing the ideal $\mathbf{a}(\theta)$ in the constraint of $(\mathcal{P} 2)$ with the measured $\mathbf{a}(\theta)$. However, this will require $(\mathcal{P} 2)$ to be solved online whenever a new set of calibration data becomes available. Notwithstanding, it will be shown in Section IV that there is no practical advantage in performing calibration.

\section{SyMMETRY PROPERTIES OF THE TRANSFORMATION MATRIX}

It is easy to see from (3) and (5) that the Davies transformation matrix $\mathbf{T}_{\mathrm{D}}$ will possess a number of symmetry properties between its rows as well as amongst the elements across a row. As it transpires, the robust transformation matrix $\mathbf{T}_{\mathrm{R}}$ that results from solving problem $(\mathcal{P} 2)$ also exhibits the same symmetry. In this section, we summarize these properties. Their proofs are given in the appendices. In the next section, we give a numerical example demonstrating how the symmetry properties can be exploited to greatly reduce the computational effort required to solve $(\mathcal{P} 2)$.

For convenience, we first renumber the rows of $\mathbf{T}_{\mathrm{R}}$ as follows.

$$
\tilde{\mathbf{t}}_{m-M_{o}-1}=\mathbf{t}_{m}, \quad m=1, \ldots, M
$$

where $M_{O}$ is given by (6). Thus, the first row of $\mathbf{T}_{\mathrm{R}}$ is $\tilde{\mathbf{t}}_{-M_{o}}(m=1)$, the last row is $\tilde{\mathbf{t}}_{M_{o}}(m=M)$, and the middle row is $\tilde{\mathbf{t}}_{0}\left(m=M_{o}+1\right)$. Likewise, we renumber the error terms and bounds on the constraints of $(\mathcal{P} 2)$, respectively, as follows:

and

$$
\tilde{e}_{m-M_{o}-1}(\theta)=e_{m}(\theta), \quad m=1, \ldots, M
$$

$$
\tilde{\varepsilon}_{m-M_{o}-1}=\varepsilon_{m}, \quad m=1, \ldots, M .
$$

The above notations lead to the following alternate statement of problem $(\mathcal{P} 2)$.
Problem (P3) : For $p=-M_{o}, \ldots, M_{o}$

$$
\min _{\tilde{\mathbf{t}}_{p}}\left\|\tilde{\mathbf{t}}_{p}\right\|_{F}^{2}
$$

subject to

$$
\begin{aligned}
& \left|\operatorname{Re}\left\{\tilde{e}_{p}(\theta)\right\}\right| \leq \tilde{\varepsilon}_{p} \quad \text { and } \\
& \left|\operatorname{Im}\left\{\tilde{e}_{p}(\theta)\right\}\right| \leq \tilde{\varepsilon}_{p}, \quad \forall \theta \in[-\pi, \pi]
\end{aligned}
$$

where, from (13) and (1) with $G_{n}(\theta)=1$

$$
\begin{aligned}
\tilde{e}_{p}(\theta) & =\tilde{\mathbf{t}}_{p}^{T} \mathbf{a}(\theta)-e^{j p \theta} \\
& =\sum_{n=1}^{N}\left[\tilde{\mathbf{t}}_{p}\right]_{n} \exp \left\{j k r \cos \left(\theta-\gamma_{n}\right)\right\}-e^{j p \theta} .
\end{aligned}
$$

Theorem 1: If $\tilde{\varepsilon}_{-p}=\tilde{\varepsilon}_{p}, p=0, \ldots, M_{o}$, then the rows of $\mathbf{T}_{\mathrm{R}}$ satisfy

$$
\tilde{\mathbf{t}}_{-p}=(-1)^{p} \tilde{\mathbf{t}}_{p}^{*}
$$

Proof: See Appendix II.

Corollary: The elements of $\tilde{\mathbf{t}}_{0}$ are real.

Theorem 1 asserts that the $(-p)$ th subproblem of $(\mathcal{P} 3)$ is solved once the $p$ th subproblem is solved. The number of subproblems in $(\mathcal{P} 3)$ that one needs to solve is thus approximately halved. The next theorem relates the symmetries that exist amongst the elements of a row of $\mathbf{T}_{R}$. This theorem is highly significant since it can greatly reduce the number of decision variables in each subproblem of $(\mathcal{P} 3)$ leading to a much reduced computational time as well as a numerically more accurate solution.

Recall the UCA has $N$ elements and is oriented with element 1 at $\theta=0^{\circ} .^{3}$

Theorem 2: The row elements of $\mathbf{T}_{\mathrm{R}}$ satisfy the following symmetry properties where \lceil\rceil and \lfloor\rfloor denote the ceiling and floor operators, respectively. For $p=0, \ldots$, $M_{o}, i=0, \ldots,(N-1)$

$$
\begin{aligned}
{\left[\tilde{\mathbf{t}}_{p}\right]\left(1+\left\lfloor\frac{i}{2}\right\rfloor-n\right) } & \bmod N+1 \\
= & e^{-j 2 p\left(1-\frac{2 i}{N}\right) \frac{\pi}{2}}\left[\tilde{\mathbf{t}}_{p}^{*}\right]_{\left(n+\left\lceil\frac{i}{2}\right\rceil-1\right) \bmod N+1}
\end{aligned}
$$

provided

$$
2 p\left(1-\frac{2 i}{N}\right) \in \mathbb{Z} .
$$

Proof: See Appendix III.

An example illustrating the application of Theorem 2 is given in the next section.

\section{NUMERICAL EXAMPLES}

\section{A. Reduction of Decision Variables}

Consider a UCA with $N=12, M=9$, and $r=0.61 \lambda$. Rows $\tilde{\mathbf{t}}_{-1}$ and $\tilde{\mathbf{t}}_{1}$ (i.e., $\mathbf{t}_{4}$ and $\mathbf{t}_{6}$ ) of the Davies transformation matrix $\mathbf{T}_{\mathrm{D}}$ of this array were found to have large norms. Accordingly, as per the design strategy described in Section II-C, we would solve $(\mathcal{P} 3)$ twice, once for $\tilde{\mathbf{t}}_{-1}$ and once for $\tilde{\mathbf{t}}_{1}$. However, Theorem 1 says we only need to solve $(\mathcal{P} 3)$ once. Suppose we choose to solve for $\tilde{\mathbf{t}}_{1}$.

\footnotetext{
${ }^{3}$ We assume element 1 is at $\theta=0^{\circ}$ with no loss of generality.
} 
From (22) of Theorem 2, we next look for $i, i \in\{0, \ldots, 11\}$, such that $2 p(1-(2 i / N))=2(1-(i / 6))$ is an integer. This gives $i=0,3,6,9$. The symmetry relations for the elements of $\tilde{\mathbf{t}}_{1}$ are thus given by

$$
\begin{array}{ll}
\underline{i=0} & {\left[\tilde{\mathbf{t}}_{1}\right]_{(1-n) \bmod 12+1}=(-1)\left[\tilde{\mathbf{t}}_{1}\right]_{(n-1) \bmod 12+1}^{*} .} \\
\underline{i=3} & {\left[\tilde{\mathbf{t}}_{1}\right]_{(2-n) \bmod 12+1}=(-j)\left[\tilde{\mathbf{t}}_{1}\right]_{(n+1) \bmod 12+1}^{*}} \\
\underline{i=6} & {\left[\tilde{\mathbf{t}}_{1}\right]_{(4-n) \bmod 12+1}=(+1)\left[\tilde{\mathbf{t}}_{1}\right]_{(n+2) \bmod 12+1}^{*}} \\
\underline{i=9} & {\left[\tilde{\mathbf{t}}_{1}\right]_{(5-n) \bmod 12+1}=(+j)\left[\tilde{\mathbf{t}}_{1}\right]_{(n+4) \bmod 12+1}^{*}}
\end{array} .
$$

Denote the $n$th element of $\tilde{\mathbf{t}}_{1}$ by $\alpha_{n}+j \beta_{n}$, where $\alpha_{n}, \beta_{n} \in \mathbb{R}$. Application of the above symmetry relations yields the structure for the row elements of $\tilde{\mathbf{t}}_{1}$ found in (27), shown at the bottom of the page. Clearly, there are only three distinct decision variables, as opposed to 24 in the original problem. Substituting (27) into $(\mathcal{P} 3)$ with $p=1$, and simplifying the expression, the problem can be rewritten as a standard quadratic semi-infinite programming problem as follows.

Problem $(\mathcal{P} 4)$ :

$$
\min _{\beta_{1}, \alpha_{2}, \beta_{2}}\left(8 \alpha_{2}^{2}+4 \beta_{1}^{2}+8 \beta_{2}^{2}\right)=\min _{\boldsymbol{\xi}} \frac{1}{2} \boldsymbol{\xi}^{T} \mathbf{Q} \xi
$$

subject to

$$
\mathbf{A}(\theta) \xi-\mathbf{c}(\theta) \leq \mathbf{0}, \quad \forall \theta \in[-\pi, \pi]
$$

where

$$
\begin{aligned}
\boldsymbol{\xi}= & {\left[\begin{array}{lll}
\alpha_{2} & \beta_{1} & \beta_{2}
\end{array}\right]^{T} \in \mathbb{R}^{3} } \\
\mathbf{Q}= & \operatorname{diag}(16,8,16) \\
\mathbf{A}(\theta)= & {\left[\begin{array}{ll}
\operatorname{Re}\{\mathbf{g}(\theta)\} & -\operatorname{Re}\{\mathbf{g}(\theta)\} \\
& \operatorname{Im}\{\mathbf{g}(\theta)\} \quad-\operatorname{Im}\{\mathbf{g}(\theta)\}
\end{array}\right]^{T} \in \mathbb{R}^{4 \times 3} } \\
\mathbf{c}(\theta)= & {\left[\begin{array}{ll}
\tilde{\varepsilon}_{1}+\cos \theta & \tilde{\varepsilon}_{1}-\cos \theta \\
& \tilde{\varepsilon}_{1}+\sin \theta \quad \tilde{\varepsilon}_{1}-\sin \theta
\end{array}\right]^{T} \in \mathbb{R}^{4} }
\end{aligned}
$$

and

$$
\begin{aligned}
g(\theta)= & {\left[\begin{array}{c}
4 \sin \left(k r \frac{1}{2} \cos \theta\right) \cos \left(k r \frac{\sqrt{3}}{2} \sin \theta\right) \\
-2 \sin (k r \cos \theta) \\
-4 \sin \left(k r \frac{\sqrt{3}}{2} \cos \theta\right) \cos \left(k r \frac{1}{2} \sin \theta\right)
\end{array}\right] } \\
& +j\left[\begin{array}{c}
4 \cos \left(k r \frac{\sqrt{3}}{2} \cos \theta\right) \sin \left(k r \frac{1}{2} \sin \theta\right) \\
-2 \sin (k r \sin \theta) \\
-4 \cos \left(k r \frac{1}{2} \cos \theta\right) \sin \left(k r \frac{\sqrt{3}}{2} \sin \theta\right)
\end{array}\right] .
\end{aligned}
$$

\section{B. Performance of the Robust Transformation Matrix With MUSIC}

In [18], it was shown that the Davies transformation can be highly susceptible to perturbations in $\mathbf{a}(\theta)$. In the next set of numerical studies, we investigate the performance of the robust transformation matrix for a UCA with radius $1.118 \lambda, N=15$, and $M=13$, and a signal scenario consisting of five fully correlated signals, each with an signal-to-noise ratio (SNR) of $10 \mathrm{~dB}$, arriving from $-150^{\circ},-100^{\circ}, 0^{\circ}, 37^{\circ}$, and $85^{\circ}$. The MUSIC algorithm was chosen to visualize the effectiveness of our proposal. Fig. 2 shows the MUSIC spectrum obtained from the UCA where the spectrum was calculated using a 200-snapshot finite-sample covariance matrix, and forward-backward spatial smoothing [4] with five subarrays, each of nine elements, was implemented on the Davies array to restore the rank of the covariance matrix. Noise prewhitening was also invoked since the noise in the Davies array is not spatially white [2]. Fig. 2 also shows the MUSIC spectra that result after the array was subjected to three independent realizations of perturbations, denoted by "Perturbed 1", "Perturbed 2," and "Perturbed 3" (see Table I). In each realization, the complex gain of each antenna element was individually perturbed from the nominal gain of 1 , its position was perturbed from its nominal position, where the perturbations in the $x$ and $y$ directions are independent, and mutual coupling between the antenna elements was introduced. Mutual coupling was assumed to be significant only for adjacent UCA elements. The steering vector of the nonideal UCA is given by $\mathbf{M} \tilde{a}(\theta)$ where

$$
\begin{aligned}
\mathbf{M}= & {\left[\begin{array}{ccccc}
1 & \delta^{(c)} & 0 & \cdots & \delta^{(c)} \\
\delta^{(c)} & \ddots & \ddots & & 0 \\
0 & \ddots & & & \vdots \\
\vdots & & & & \delta^{(c)} \\
\delta^{(c)} & 0 & \cdots & \delta^{(c)} & 1
\end{array}\right] } \\
\delta^{(c)}= & \delta^{\left(c_{1}\right)}+j \delta^{\left(c_{2}\right)} \\
{[\tilde{\mathbf{a}}(\theta)]_{n}=} & \left(1+\delta_{n}^{\left(g_{1}\right)}+j \delta_{n}^{\left(g_{2}\right)}\right) \\
& \times \exp \left\{j k r \left[\cos \theta\left(\cos \gamma_{n}+\delta_{n}^{(x)}\right)\right.\right. \\
& \left.\left.\quad+\sin \theta\left(\sin \gamma_{n}+\delta_{n}^{(y)}\right)\right]\right\} .
\end{aligned}
$$

$\mathbf{M}$ is the mutual coupling matrix with complex perturbations $\delta^{(c)}, \delta_{n}^{\left(g_{1}\right)}+j \delta_{n}^{\left(g_{2}\right)}$ is the perturbations on the complex gain, and $\left(\delta_{n}^{(x)}, \delta_{n}^{(y)}\right)$ gives the perturbations (relative to $r$ ) in the $x$ and $y$ direction of the $n$th UCA element. The distributions of the perturbations are as follows:

$$
\begin{aligned}
\delta_{n}^{\left(g_{1}\right)}, \delta_{n}^{\left(g_{2}\right)} \sim \mathcal{N}(0,0.02), \quad \delta_{n}^{(x)}, \delta_{n}^{(y)} \sim \mathcal{N}(0,0.005), \\
\delta^{\left(c_{1}\right)}, \delta^{\left(c_{2}\right)} \sim \mathcal{N}(0,0.01) .
\end{aligned}
$$

Clearly, the MUSIC spectra of the perturbed arrays in Fig. 2, with missed and false peaks, are unacceptable. It may be surprising that the ideal UCA also failed to work correctly in this scenario. This can be attributed to the fact that the finite-sample covariance matrix, rather than the exact covariance matrix, was used in the computation of the MUSIC spectra. The difference between these two matrices resembles a perturbation of the UCA which is amplified by the large row norms in the Davies matrix. It can be shown that MUSIC can accurately resolve the signals if the exact covariance matrix is used, although in practice it is almost impossible to obtain a perfect estimate of such a matrix.

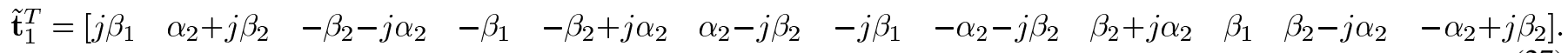


TABLE I

THREE INDEPENDENT REALIZATIONS OF RANDOM PERTURBATIONS ON THE UCA

\begin{tabular}{|c||c|c|c|c|c|c|}
\hline & \multicolumn{2}{|c|}{ Perturbed 1 $\left(\times 10^{-2}\right)$} & \multicolumn{2}{c|}{ Perturbed 2 $\left(\times 10^{-2}\right)$} & \multicolumn{2}{c|}{ Perturbed 3 $\left(\times 10^{-2}\right)$} \\
\hline & \multicolumn{2}{|c|}{$\delta^{\left(c_{1}\right)}+j \delta^{\left(c_{2}\right)}$} & \multicolumn{2}{|c|}{$\delta^{\left(c_{1}\right)}+j \delta^{\left(c_{2}\right)}$} & \multicolumn{2}{|c|}{$\delta^{\left(c_{1}\right)}+j \delta^{\left(c_{2}\right)}$} \\
\hline \hline & \multicolumn{2}{|c|}{$0.879-j 0.723$} & \multicolumn{2}{|c|}{$1.051-j 0.308$} & \multicolumn{2}{c|}{$0.714+j 0.117$} \\
\hline $\mathbf{n}$ & $\delta_{n}^{\left(g_{1}\right)}+j \delta_{n}^{\left(g_{2}\right)}$ & $\left(\delta_{n}^{(x)}, \delta_{n}^{(y)}\right)$ & $\delta_{n}^{\left(g_{1}\right)}+j \delta_{n}^{\left(g_{2}\right)}$ & $\left(\delta_{n}^{(x)}, \delta_{n}^{(y)}\right)$ & $\delta_{n}^{\left(g_{1}\right)}+j \delta_{n}^{\left(g_{2}\right)}$ & $\left(\delta_{n}^{(x)}, \delta_{n}^{(y)}\right)$ \\
\hline $\mathbf{1}$ & $2.896+j 1.204$ & $(-1.732,0.312)$ & $3.348+j 2.235$ & $(0.383,-0.429)$ & $2.495+j 0.086$ & $(0.072,-0.411)$ \\
\hline $\mathbf{2}$ & $-0.889+j 2.203$ & $(-0.511,0.054)$ & $0.256-j 1.802$ & $(0.003,-0.003)$ & $0.675+j 0.128$ & $(0.764,-0.267)$ \\
\hline $\mathbf{3}$ & $0.536-j 0.050$ & $(0.616,-0.005)$ & $-2.034+j 0.762$ & $(-0.012,0.214)$ & $-1.521-j 0.494$ & $(-0.359,1.365)$ \\
\hline $\mathbf{4}$ & $0.238+j 0.085$ & $(-0.430,-0.568)$ & $-0.515-j 1.042$ & $(0.482,-0.633)$ & $0.190+j 1.531$ & $(0.614,-0.687)$ \\
\hline $\mathbf{5}$ & $-0.230-j 0.762$ & $(0.651,0.659)$ & $0.236+j 2.030$ & $(0.280,-0.349)$ & $-0.066-j 1.418$ & $(0.238,-0.349)$ \\
\hline $\mathbf{6}$ & $0.556+j 0.931$ & $(-0.258,0.442)$ & $-0.994-j 0.544$ & $(-0.537,0.565)$ & $2.627+j 1.415$ & $(0.681,0.382)$ \\
\hline $\mathbf{7}$ & $-2.117+j 0.158$ & $(0.760,0.352)$ & $0.085-j 5.614$ & $(0.056,-0.135)$ & $-0.607-j 1.492$ & $(-0.469,-0.125)$ \\
\hline $\mathbf{8}$ & $1.199-j 1.896$ & $(0.838,0.368)$ & $0.614-j 2.595$ & $(0.227,0.978)$ & $1.975+j 3.089$ & $(0.335,0.279)$ \\
\hline $\mathbf{9}$ & $2.213-j 0.779$ & $(-0.064,0.232)$ & $0.414-j 2.010$ & $(0.214,-0.710)$ & $-3.758-j 1.459$ & $(-0.066,-0.014)$ \\
\hline $\mathbf{1 0}$ & $2.156-j 4.060$ & $(-0.357,-0.614)$ & $-1.631-j 2.370$ & $(-0.108,0.176)$ & $-0.657+j 0.340$ & $(-0.418,-0.018)$ \\
\hline $\mathbf{1 1}$ & $0.048-j 3.236$ & $(-0.291,0.513)$ & $1.432+j 0.075$ & $(0.284,-0.657)$ & $1.003-j 0.388$ & $(-0.274,-0.884)$ \\
\hline $\mathbf{1 2}$ & $-0.283-j 2.823$ & $(0.018,-0.521)$ & $-3.263+j 0.112$ & $(0.374,0.246)$ & $-1.496+j 0.545$ & $(-0.088,0.543)$ \\
\hline $\mathbf{1 3}$ & $-2.399+j 2.275$ & $(0.567,0.421)$ & $2.679+j 1.102$ & $(-0.163,-0.521)$ & $-0.081-j 2.157$ & $(-0.160,-0.324)$ \\
\hline $\mathbf{1 4}$ & $-2.332-j 0.620$ & $(0.367,0.085)$ & $-0.596+j 3.572$ & $(-0.176,0.025)$ & $3.865+j 0.155$ & $(-0.459,0.136)$ \\
\hline $\mathbf{1 5}$ & $-0.288-j 1.723$ & $(-0.810,0.017)$ & $3.034+j 1.740$ & $(0.490,0.687)$ & $0.973+j 0.801$ & $(-0.049,-0.127)$ \\
\hline
\end{tabular}

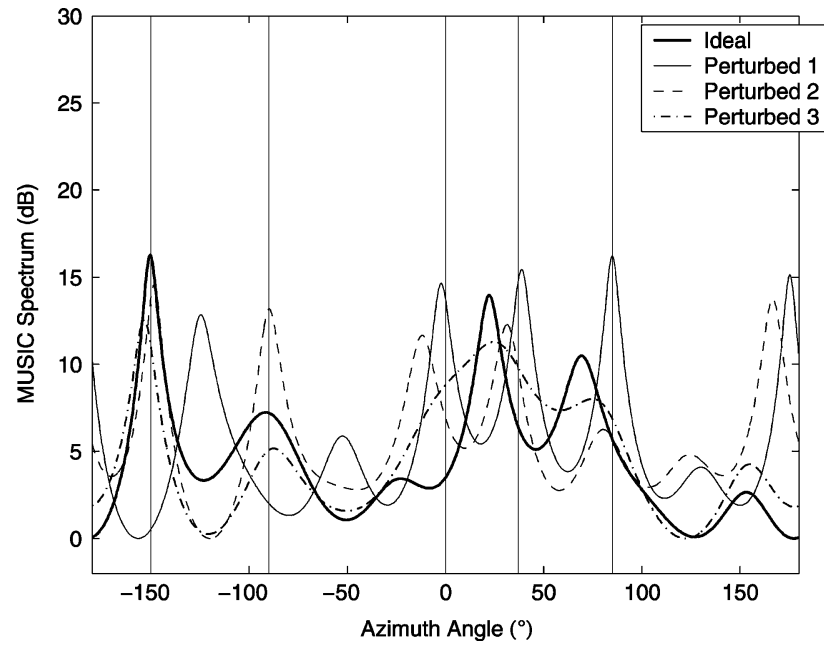

Fig. 2. MUSIC spectra of ideal UCA and three perturbed UCAs with the Davies matrix and finite-sample covariance matrix.

Table II summarizes the squared-norm and maximum real and imaginary errors of each row of the Davies matrix. As can be seen, the squared-norms of rows 6 and 8 greatly exceed the robustness criterion of $N / M=15 / 13=1.15$.

To derive the robust transformation matrix $\mathbf{T}_{R}$, we replace rows 6 and 8 of the Davies matrix by rows found by solving $(\mathcal{P} 3)$ with $\tilde{\varepsilon}_{1}$ set to 0.7 . The MUSIC spectra obtained from $\mathbf{T}_{\mathrm{R}}$ are shown in Fig. 3 where the perturbed arrays are the same as those in Fig. 2. The characteristics of $\mathbf{T}_{\mathrm{R}}$ are also summarized in Table II. Note the increase in approximation error in rows 6 and 8. Note too that although the norm of rows 6 and 8 of $\mathbf{T}_{R}$ is still much greater than the recommended $N / M=1.15, \mathbf{T}_{\mathrm{R}}$ appears to perform satisfactorily. This suggests the $N / M$ criterion is overly conservative.

Next, we assume the array was perturbed but calibration data are available for the first perturbed array, i.e., "Perturbed 1" of Table I and Figs. 2 and 3. This allows us to apply a least squares fit as in [2] to derive the calibrated Davies transformation matrix.
TABLE II

CHARACTERISTICS OF THE DAVIES AND RoBUSt TRANSFORMATION MATRICES FOR $N=15, M=13$, AND $r=1.118 \lambda$

\begin{tabular}{|c|c|c|c|c|}
\hline & \multicolumn{2}{|c|}{ Davies Matrix $\mathbf{T}_{\mathrm{D}}$} & \multicolumn{2}{c|}{ Robust Matrix $\mathbf{T}_{\mathrm{R}}$} \\
\hline Row \# & Squared-norm & Max Error & Squared-norm & Max Error \\
\hline $\mathbf{1 , 1 3}$ & 0.574 & 0.1770 & & \\
\hline $\mathbf{2 , 1 2}$ & 0.559 & 0.0701 & & \\
\hline $\mathbf{3 , 1 1}$ & 2.923 & 0.0570 & & \\
\hline $\mathbf{4 , 1 0}$ & 2.214 & 0.0159 & & \\
\hline $\mathbf{5 , 9}$ & 0.745 & 0.0027 & & \\
\hline $\mathbf{6 , 8}$ & 6513.34 & 0.0710 & 148.60 & 0.7030 \\
\hline $\mathbf{7}$ & 0.740 & 0.0000 & & \\
\hline
\end{tabular}

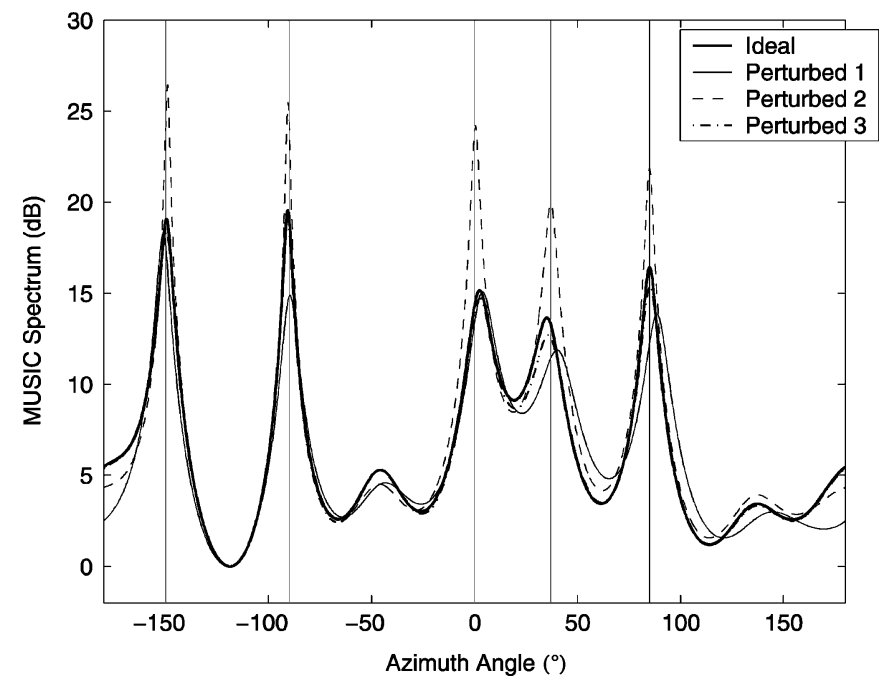

Fig. 3. MUSIC spectra of ideal UCA and three perturbed UCAs with robust transformation matrix and finite-sample covariance matrix.

In our application of the least-squares fit, we assumed perfect calibration data were available for "Perturbed 1" where the calibration was performed over a uniform grid spacing of $5^{\circ}$. Fig. 4 shows the MUSIC spectrum obtained for the calibrated array. As can be seen, the calibrated array failed to resolve all signals, for the same reason as the ideal case of Fig. 2, except that here the robustness problem is compounded by the large approximation 


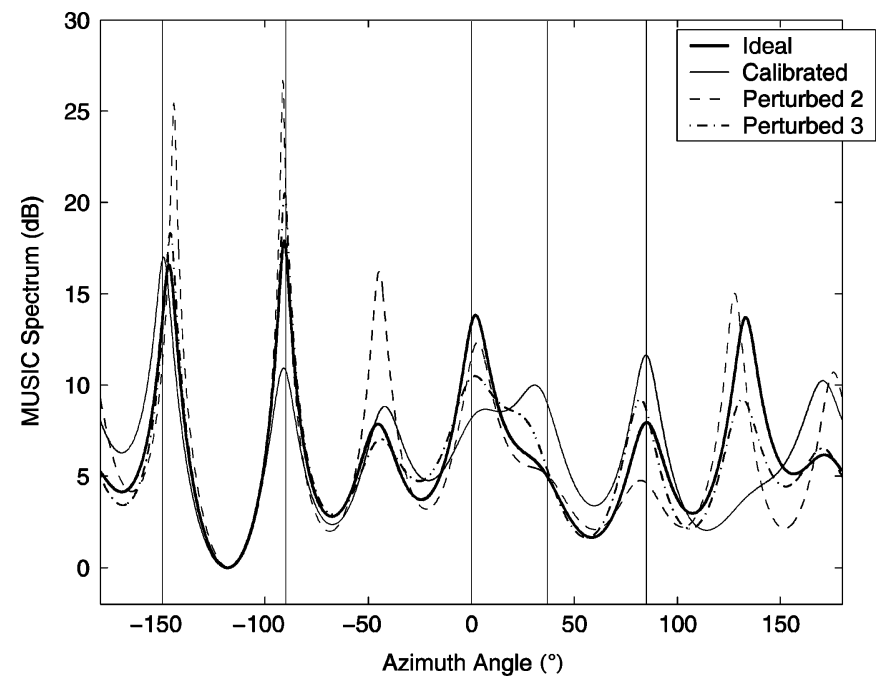

Fig. 4. MUSIC spectra of the four UCAs with Davies transformation matrix calibrated against the first perturbed UCA and finite-sample covariance matrix.

TABLE III

CHARACTERISTICS OF THE CALIBRATED DAVIES AND RoBUST TRANSFORMATION MATRICES FOR A NONIDEAL UCA WITH $N=15, M=13$ AND $r=1.118 \lambda$

\begin{tabular}{|c|c|c|c|c|}
\hline & \multicolumn{2}{|c|}{ Calibrated Davies Matrix } & \multicolumn{2}{c|}{$\begin{array}{c}\text { Calibrated Robust Matrix } \\
\mathbf{T}_{\mathrm{D}} \mathbf{C}\end{array}$} \\
\hline Row \# & Squared-norm & Max Error & Squared-norm & Max Error \\
\hline $\mathbf{1}$ & 6.856 & 0.2410 & 1.003 & 0.1899 \\
\hline $\mathbf{2}$ & 1.065 & 0.0889 & 1.135 & 0.0870 \\
\hline $\mathbf{3}$ & 4.024 & 0.0845 & 4.318 & 0.0850 \\
\hline $\mathbf{4}$ & 4.706 & 0.0565 & 5.587 & 0.0534 \\
\hline $\mathbf{5}$ & 1.038 & 0.0146 & 1.069 & 0.0184 \\
\hline $\mathbf{6}$ & 1254.649 & 0.7895 & 52.165 & 0.8228 \\
\hline $\mathbf{7}$ & 1.003 & 0.0215 & 1.198 & 0.0270 \\
\hline $\mathbf{8}$ & 1258.540 & 0.7988 & 53.039 & 0.8220 \\
\hline $\mathbf{9}$ & 1.046 & 0.0146 & 1.081 & 0.0178 \\
\hline $\mathbf{1 0}$ & 4.732 & 0.0556 & 5.582 & 0.0530 \\
\hline $\mathbf{1 1}$ & 4.019 & 0.0854 & 4.291 & 0.0852 \\
\hline $\mathbf{1 2}$ & 1.022 & 0.0897 & 1.144 & 0.0872 \\
\hline $\mathbf{1 3}$ & 7.031 & 0.2416 & 1.009 & 0.1899 \\
\hline
\end{tabular}

errors (see Table III ${ }^{4}$ ). In addition, as can be seen from Table III, the transformation matrix of the calibrated array also has large row norms. Therefore, if the perturbations are time-varying such that the calibrated array is perturbed further to, say, "Perturbed 2" or "Perturbed 3," then MUSIC will also suffer severe degradation, as can be seen from Fig. 4. Likewise, the performance of the "Ideal" array also degrades severely since in this scenario, the "Ideal" array can be regarded as just another perturbation from the calibrated array. An alternative interpretation of the above result is that, suppose calibration data were corrupted by measurement errors, then severe degradation in performance can also result.

Finally, we follow Remark 2 of Section II-D and combine the robust transformation matrix with the calibrated array transformation matrix of "Perturbed 1" (see Table III) such that the transformation matrix is now $\mathbf{T}=\mathbf{T}_{\mathrm{R}} \mathbf{C}$. The calibrated matrix is found to be well conditioned. Fig. 5 shows the MUSIC spectra obtained from the same set of arrays as in Figs. 2-4 but with the

\footnotetext{
${ }^{4}$ We show all rows since, due to the lack of symmetry in the perturbed UCA, the rows of the transformation matrix will not have the symmetry properties outlined in Section IV.
}

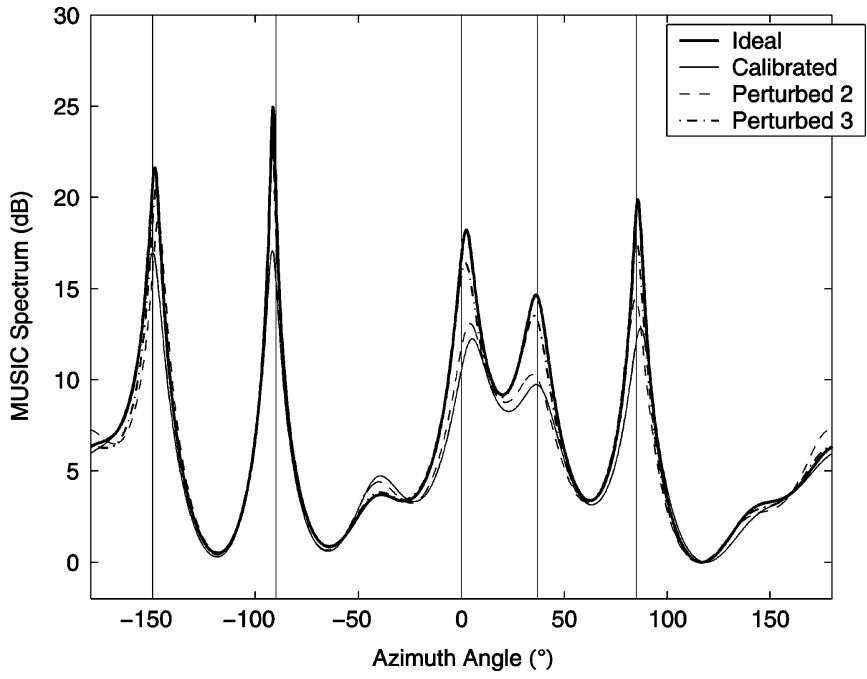

Fig. 5. MUSIC spectra of the four UCAs with transformation matrix calibrated against the first perturbed UCA and made robust, and with finite-sample covariance matrix.

calibrated robust matrix. Comparing Figs. 4 and 5, it is clear that the calibration step of [2] does not yield a robust transformation matrix, while the calibrated robust matrix can perform satisfactorily. In addition, comparing Figs. 3 and 5, we observe that the robust matrix will perform just as well without calibration. However, the requirement for $\mathbf{C}$ to be well conditioned restricts its application in general. Moreover, incorporating $\mathbf{C}$ can add a degree of uncertainty into the performance, since as compared with the robust matrix $\mathbf{T}_{\mathrm{R}}$, the row norms of the calibrated matrix $\mathbf{T}$ can increase from the use of $\mathbf{C}$ even when $\mathbf{C}$ is well conditioned (compare Tables II and III). For these reasons, we shall omit the calibration procedure when determining $\mathbf{T}_{R}$.

\section{RMSE Performance of the Robustness Transformation Matrix With Root-WSF}

While the MUSIC spectra provide a good visualization of the problem with robustness in the Davies transformation and the effectiveness of our proposal to mitigate this problem, they do not give any statistical performance. In the following examples, we evaluate the RMSE performances of both the Davies and the robust matrices with respect to the root-WSF algorithm [24]. Root-WSF has a maximum likelihood (ML)-like performance in correlated signal environments, and like MUSIC with spatial smoothing, also requires Vandermonde form in the array steering vector.

Scenario 1: RMSE Versus Angular Separation without a Large Norm in $\mathbf{T}_{\mathrm{D}}$ : As a point of reference, we begin with a UCA with $r=0.721 \lambda, N=15$, and $M=13$. This gives a well-behaved Davies matrix, as can be seen from Table IV. The squared norm of rows 1 and 13 (i.e., 8.802 79) is larger than $N / M=1.15$, it is still at the same order of magnitude. Hence, the UCA is robust and only a small performance improvement can be obtained via the proposed procedure. In this scenario, three fully correlated signals (with correlation coefficient of 1 ), each with SNR of $0 \mathrm{~dB}$, were present. The DOAs of two of the signals were fixed to $-60^{\circ}$ and $0^{\circ}$, while the third was allowed to step from $30^{\circ}$ to $60^{\circ}$, in increments of $2.5^{\circ}$. The number of 
TABLE IV

CHARACTERISTICS OF THE DAVIES AND ROBUST TRANSFORMATION MATRICES FOR $N=15, M=13$, AND $r=0.721 \lambda$

\begin{tabular}{|c||c|c||}
\hline & \multicolumn{2}{|c|}{ Davies Matrix } \\
\hline Row \# & Squared-norm & $\begin{array}{c}\text { Max Error } \\
\left(\times 10^{-2}\right)\end{array}$ \\
\hline \hline $\mathbf{1 , 1 3}$ & 8.80279 & 2.95268 \\
\hline $\mathbf{2 , 1 2}$ & 1.68193 & 0.30749 \\
\hline $\mathbf{3 , 1 1}$ & 0.53753 & 0.03732 \\
\hline $\mathbf{4 , 1 0}$ & 0.37360 & 0.00608 \\
\hline $\mathbf{5 , 9}$ & 1.55657 & 0.00223 \\
\hline $\mathbf{6 , 8}$ & 1.15926 & 0.00033 \\
\hline $\mathbf{7}$ & 0.68155 & 0.00008 \\
\hline
\end{tabular}

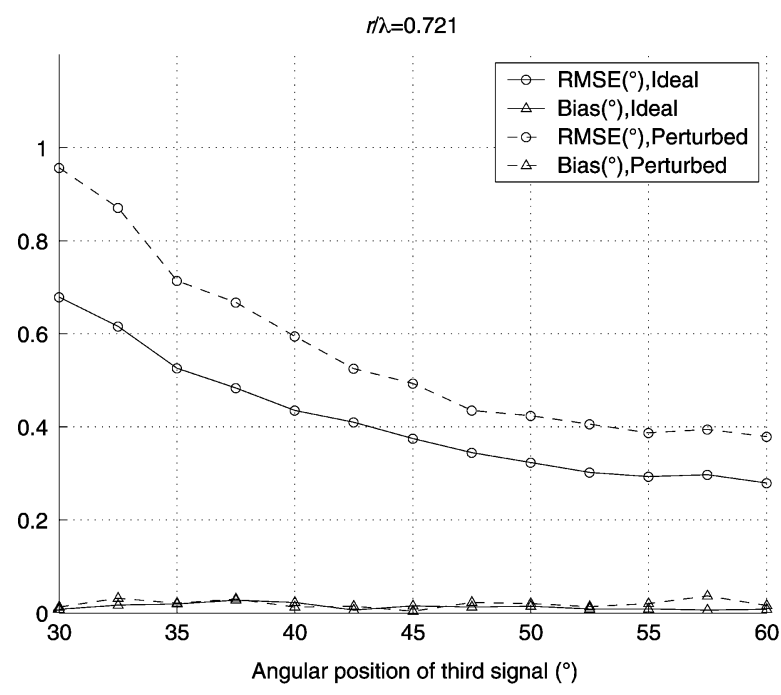

$r / \lambda=0.818$

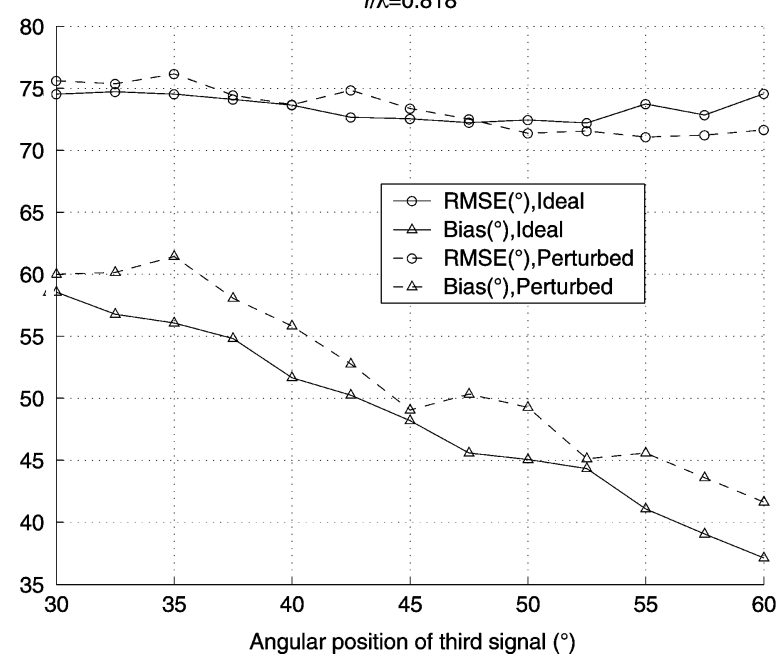

Fig. 6. Average RMSE and bias performances of DOA estimation algorithms on the Davies array versus angular position of the third signal, for two different $r / \lambda$ 's.

snapshots $K$ was fixed at 200 and 500 Monte Carlo trials were conducted. The UCA was subjected to the same perturbation statistics as given in Section IV-B. The RMSE and bias results are shown in Fig. 6 and will be discussed together with the results of Scenario 2.

Scenario 2: RMSE Versus Angular Separation With a Large Norm in $\mathbf{T}_{\mathrm{D}}$ : Scenario 1 is repeated for $r=0.818 \lambda$ and the
TABLE V

CHARACTERISTICS OF THE DAVIES TRANSFORMATION MATRICES FOR $N=15, M=13$, AND $r=0.818 \lambda$

\begin{tabular}{|c||c|c||c|c||}
\hline \multicolumn{1}{|||}{} & \multicolumn{2}{|c||}{ Davies Matrix $(r=0.818 \lambda)$} & \multicolumn{2}{c|}{ Robust Matrices (I) and (II) } \\
\hline \hline Row \# & Squared-norm & $\begin{array}{c}\text { Max Error } \\
\left(\times 10^{-2}\right)\end{array}$ & Squared-norm & Max Error \\
\hline $\mathbf{1 , 1 3}$ & 3.14200 & 4.66128 & & \\
\hline $\mathbf{2 , 1 2}$ & 0.85806 & 0.66800 & & \\
\hline $\mathbf{3 , 1 1}$ & 0.42321 & 0.11560 & & \\
\hline $\mathbf{4 , 1 0}$ & 0.57908 & 0.03028 & & \\
\hline $\mathbf{5 , 9}$ & 168062.2 & 3.35111 & (I) 19.462 & (I) 0.8593 \\
& & & & (II) 2.087 \\
\hline $\mathbf{6 , 8}$ & 0.57741 & 0.00121 & & \\
\hline $\mathbf{7}$ & 3.84654 & 0.00011 & & \\
\hline
\end{tabular}

results shown in Table V and Fig. 6. As observed in Table V, the squared norm for rows 5 and 9 of the new $\mathbf{T}_{\mathrm{D}}$ is very large, viz. 168 062.2. It should be clear from Fig. 6 that although the introduction of array perturbations can degrade significantly the RMSE performance in Scenario $1(r=0.721 \lambda)$, all three signals were successfully resolved by both the ideal and nonideal UCAs. In addition, the biases are noticeably small. On the other hand, the RMSE and bias performances of Scenario 2 (see Fig. 6, $r=0.818 \lambda$ ) reveal that the large row norms can cause root-WSF to fail, regardless of whether array perturbations exist. The reason for this is because, with 200 snapshots, the estimated covariance matrix will differ from the exact covariance matrix and this difference is amplified by the large row norm of $\mathbf{T}_{\mathrm{D}}$. In fact, it can be verified that when the exact covariance matrix is used, the bias of root-WSF for the ideal UCA is very small-in the order of $0.001^{\circ}$.

Scenario 3: RMSE Versus Angular Separation for Robust Matrices: Scenario 2 was repeated with the robust matrices shown in Table V. Two robust matrices, with row norms of 19.462 (denoted by I) and 2.087 (denoted by II), were generated for comparisons. The RMSE and bias performances are given in Fig. 7. It is clear by comparing Figs. 6 and 7 that the proposed procedure is able to improve significantly the RMSE performances of the DOA estimator for the $r=0.818 \lambda$ array. For larger angles of the third signal, (I) achieves lower RMSE than (II), though (I) has a poorer threshold performance than (II). In general, the RMSE of (II) is dominated by the bias component, while that of (I) is dominated by the variance. This can be understood from the larger norms of (I) giving a larger variance in the estimate, yet a better Vandermonde approximation, which implies a smaller bias.

Scenario 4: RMSE Versus SNR for Robust Matrices: In the fourth scenario (see Fig. 7), the three signals were fixed at $-60^{\circ}$, $0^{\circ}$, and $40^{\circ}$, and the SNR was varied between $-10 \mathrm{~dB}$ and $10 \mathrm{~dB}$, in steps of $1 \mathrm{~dB}$. All other parameters were kept the same as in Scenario 3. It appears that the RMSE performances of root-WSF stayed approximately the same for larger SNRs. This is not surprising since the largest sources of error are array perturbations and the transformation error introduced by the proposed procedure. While a higher SNR can improve the estimate of the covariance matrix, it does not reduce these errors. As in Scenario 3, it is observed that the RMSEs of (II) are generally poorer than (I) and are dominated by the bias component, while those of (I) are dominated by the variance. However, (I) has a lower SNR threshold performance as compared with (II). 

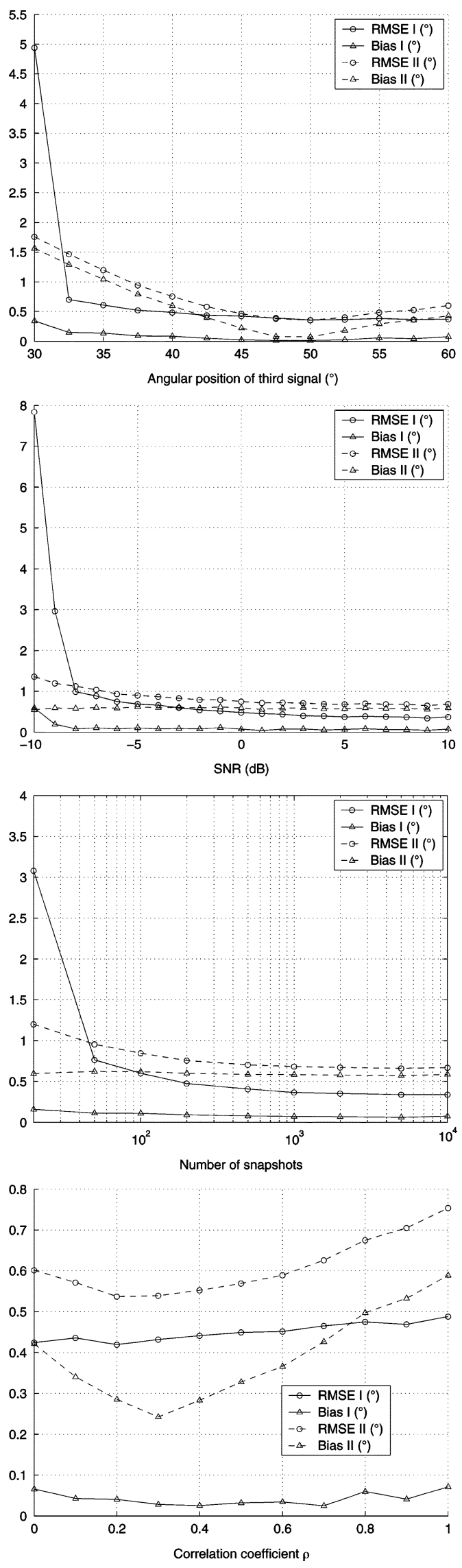

Fig. 7. Average RMSE and bias performances of DOA estimation algorithms using the robust matrix versus angular position of the third signal, SNR, number of snapshots, and correlation coefficient.
Scenario 5: RMSE Versus Number of Snapshots for Robust Matrices: The fifth scenario shown in Fig. 7 follows from Scenario 4, except that the SNRs were fixed at $0 \mathrm{~dB}$, and the number of snapshots was varied from 20 to 10000 . As in the case of varying SNRs, increasing the number of snapshots does not have a significant impact on the RMSE performance for the same underlying reason that the model errors are fixed for a given Monte Carlo trial. The same trend as in Scenarios 3 and 4 is also seen here when comparing between (I) and (II).

Scenario 6: RMSE Versus Correlation Coefficient for Robust Matrices: The sixth scenario shown in Fig. 7 set the two signals at $0^{\circ}$ and $40^{\circ}$ to be fully correlated (with correlation coefficient of 1), while the third signal at $-60^{\circ}$ had a different correlation coefficient $\rho$ with the pair of fully correlated signals where $\rho \in$ $[0,1]$ and $\rho$ is varied in steps of 0.1 . All other parameters are kept the same as in Scenario 5. Here, the impact of correlation is clearly seen in (I) where the performance degrades with an increase in correlation. The same trend can also be seen in (II), even though the poor bias performance appears to dominate the RMSEs for small correlation values (of less than 0.3).

Scenarios 2 to 6 above with $r=0.818 \lambda$ involve a pair of rows of very large norms. Thus, the proposed procedure can mean the difference between success and failure in DOA estimation. However, in a general situation where the row norms are of moderate values, the performance improvement may not be as dramatic. Moreover, since the sacrifice in transformation error can worsen the performance of DOA estimation with respect to certain parameters such as signal separation, careful consideration is necessary when applying the proposed procedure to obtain an overall improvement in performance (e.g., RMSE) with respect to all parameters (or at least, to those of interest). This has been demonstrated in the comparison between different target row norms (I) and (II). As such, the rough guide for the target row norms described earlier, while instructive of the imperfection noise amplification, is incomprehensive. Instead, an ad hoc trial and error procedure that checks the performance (of DOA estimation) over a range of row norms is more appropriate to optimize the robustness design.

Finally, we remark first that after testing with different array geometries, the dual parametrization procedure was found to be reliable and that it converges to the optimal solution within a few minutes with MATLAB on a 1-GHz Pentium IV PC. In any event, the computational time is not a major concern since $\mathbf{T}_{R}$ is computed offline. Second, as with the Davies matrix $\mathbf{T}_{\mathrm{D}}$, the robust transformation matrix $\mathbf{T}_{\mathrm{R}}$ induces a bias in the DOA estimates. This bias is unavoidable and is due to the approximation error between the steering vector of the virtual array and its desired Vandemonde form.

\section{CONCLUSION}

In this paper, we addressed the important problem of direction finding with simple DOA estimation algorithms and with nonideal UCAs in correlated signal environments. We showed that a crucial step in the solution is to find a robust transformation matrix to transform the steering vector of the UCA to one with Vandermonde form. The robust matrix is found by posing and solving a quadratic semi-infinite optimization problem, which 
trades off the Vandermonde approximation error with a matrix of lower norm. We showed that, by an appropriate formulation, we can decompose the problem into a set of much simpler optimization problems which can then be solved efficiently using the dual parametrization method of [22], [23]. Each subproblem yields a row of the robust transformation matrix. We showed further that the robust transformation matrix possesses a number of symmetrical properties between its rows and across the elements of its rows. These symmetries allow us to increase further the computational efficiency of the dual parametrization algorithm as well as the numerical accuracy of the optimum solution. Finally, we showed that we can control through the optimization parameter $\varepsilon_{m}$, a tradeoff between bias and variance of the DOA estimates. The robustness and effectiveness of the new transformation matrix is demonstrated through a series of numerical examples.

\section{APPENDIX I \\ DUAL PARAMETRIZATION METHOD}

We summarize here the dual parametrization method of [22] and [23] as applied to solve $(\mathcal{P} 2),(\mathcal{P} 3)$, and $(\mathcal{P} 4)$. Note that, for the sake of generality in presentation, we adopt in this Appendix a set of notations that differ somewhat from the remainder of this paper. In Section $\mathrm{C}$ of this appendix, we provide the mapping between the notations of this Appendix and those of problems $(\mathcal{P} 3)$ and $(\mathcal{P} 4)$.

\section{A. Problem Statement}

Consider the following quadratic semi-infinite programming problem.

\section{Problem $(\mathcal{P} 5)$ :}

$$
\min _{\mathbf{u}} \frac{1}{2} \mathbf{u}^{H} \mathbf{R u}
$$

subject to

$$
\left|\mathbf{a}^{T}(\theta) \mathbf{u}-b(\theta)\right|_{\mathbb{C}} \leq \varepsilon, \quad \forall \theta \in\left[\theta_{a}, \theta_{b}\right]
$$

where $\mathbf{u} \in \mathbb{C}^{N}, \mathbf{R} \in \mathbb{C}^{N \times N}$ is positive definite, $\mathbf{a}(\theta) \in$ $\mathbb{C}^{N}, b(\theta) \in \mathbb{C}, \varepsilon \in \mathbb{R}_{+}$, and

$$
\begin{array}{r}
\left|\mathbf{a}^{T}(\theta) \mathbf{u}-b(\theta)\right| \mathbb{C}=\max \left\{\left|\operatorname{Re}\left[\mathbf{a}^{T}(\theta) \mathbf{u}-b(\theta)\right]\right|,\right. \\
\left.\left|\operatorname{Im}\left[\mathbf{a}^{T}(\theta) \mathbf{u}-b(\theta)\right]\right|\right\} .
\end{array}
$$

Problem $(\mathcal{P} 5)$ can be rewritten as follows.

Problem $(\mathcal{P} 6)$ :

$$
\min _{\mathbf{x}} \frac{1}{2} \mathbf{x}^{T} \mathbf{Q x}
$$

subject to

$$
\mathbf{A}(\theta) \mathbf{x}-\mathbf{b}(\theta) \leq \mathbf{0}, \quad \forall \theta \in\left[\theta_{a}, \theta_{b}\right]
$$

where

$$
\begin{aligned}
& \mathbf{x}=\left[\operatorname{Re}\left[\mathbf{u}^{T}\right]\right. \\
& \left.\mathbf{I m}\left[\mathbf{u}^{T}\right]\right]^{T} \in \mathbb{R}^{2 N} \\
& \mathbf{Q}=\left[\begin{array}{cc}
\operatorname{Re}[\mathbf{R}] & -\operatorname{Im}[\mathbf{R}] \\
\operatorname{Im}[\mathbf{R}] & \operatorname{Re}[\mathbf{R}]
\end{array}\right] \in \mathbb{R}^{2 N \times 2 N}
\end{aligned}
$$

$$
\mathbf{A}(\theta)=\left[\begin{array}{cc}
\operatorname{Re}\left\{\mathbf{a}^{T}(\theta)\right\} & -\operatorname{Im}\left\{\mathbf{a}^{T}(\theta)\right\} \\
-\operatorname{Re}\left\{\mathbf{a}^{T}(\theta)\right\} & \operatorname{Im}\left\{\mathbf{a}^{T}(\theta)\right\} \\
\operatorname{Im}\left\{\mathbf{a}^{T}(\theta)\right\} & \operatorname{Re}\left\{\mathbf{a}^{T}(\theta)\right\} \\
-\operatorname{Im}\left\{\mathbf{a}^{T}(\theta)\right\} & -\operatorname{Re}\left\{\mathbf{a}^{T}(\theta)\right\}
\end{array}\right] \in \mathbb{R}^{4 \times 2 N}
$$

and

$$
\begin{aligned}
& \mathbf{b}(\theta)=[\varepsilon+\operatorname{Re}\{b(\theta)\} \quad \varepsilon-\operatorname{Re}\{b(\theta)\} \\
& \varepsilon+\operatorname{Im}\{b(\theta)\} \quad \varepsilon-\operatorname{Im}\{b(\theta)\}]^{T} \in \mathbb{R}^{4} .
\end{aligned}
$$

\section{B. The Method}

We describe now the dual parametrization method to solve $(\mathcal{P} 6)$ [25]. Consider first the parametrized dual problem with $k$ index parameters.

Problem $(\mathcal{P} 7 k)$ :

$$
\min _{\mathbf{x}, \boldsymbol{\lambda}^{(k)}, \boldsymbol{\theta}^{(k)}} \frac{1}{2} \mathbf{x}^{T} \mathbf{Q x}+\sum_{j=1}^{k} \mathbf{b}^{T}\left(\theta_{j}\right) \boldsymbol{\lambda}_{j}
$$

subject to

$$
\begin{aligned}
& \mathbf{Q x}+\sum_{j=1}^{k} \mathbf{A}^{T}\left(\theta_{j}\right) \boldsymbol{\lambda}_{j}=\mathbf{0}, \quad \boldsymbol{\lambda}_{j} \geq \mathbf{0} \quad \text { and } \\
& \theta_{a} \leq \theta_{j} \leq \theta_{b}, \quad j=1, \ldots, k
\end{aligned}
$$

where $\lambda_{j} \in \mathbb{R}^{4}, \lambda^{(k)}=\left[\begin{array}{llll}\lambda_{1}^{T} & \lambda_{2}^{T} & \cdots & \lambda_{k}^{T}\end{array}\right]^{T}$, and $\boldsymbol{\theta}^{(k)}=$ $\left[\begin{array}{llll}\theta_{1} & \theta_{2} & \cdots & \theta_{k}\end{array}\right]^{T}$. The main results relating $(\mathcal{P} 6)$ and $(\mathcal{P} 7 k)$ are stated in the following theorem [22].

\section{Theorem A1:}

a) There exists a $k^{*}$ satisfying $0 \leq k^{*} \leq 2 N$ such that the optimal value sequence $\{V(\mathcal{P} 7 k)\}_{k=1}^{k^{*}}$ decreases strictly, and for $k>k^{*}, V(\mathcal{P} 7 k)=V\left(\mathcal{P} 7 k^{*}\right)$.

b) The number $k^{*}$ in a) is the smallest whole number such that for any $k \geq k^{*}$, the global solution of $(\mathcal{P} 7 k)$ provides the solution of $(\mathcal{P} 6)$ in the sense that, if $\left(\mathbf{x}^{*}, \boldsymbol{\lambda}^{*}, \boldsymbol{\theta}^{*}\right)$ is a solution of $(\mathcal{P} 7 k), k \geq k^{*}$, then $\mathrm{x}^{*}$ is the solution of $(\mathcal{P} 6)$.

Based on Theorem A1, [23] develops the following algorithm. Define first the following problem.

$\operatorname{Problem}(\mathcal{P} 8 \boldsymbol{k})$ : For a given $k$ and $\boldsymbol{\theta}^{(k)}=\left[\begin{array}{llll}\theta_{1} & \theta_{2} & \cdots & \theta_{k}\end{array}\right]^{T}$

$$
\min _{\mathbf{x}, \boldsymbol{\lambda}^{(k)}} \frac{1}{2} \mathbf{x}^{T} \mathbf{Q} \mathbf{x}+\sum_{j=1}^{k} \mathbf{b}^{T}\left(\theta_{j}\right) \lambda_{j}
$$

subject to

$$
\mathbf{Q x}+\sum_{j=1}^{k} \mathbf{A}^{T}\left(\theta_{j}\right) \boldsymbol{\lambda}_{j}=\mathbf{0} \quad \text { and } \quad \boldsymbol{\lambda}_{j} \geq \mathbf{0}, \quad j=1, \ldots, k .
$$

The algorithm is given by the following sequence of steps.

Step 1) Initialization. Choose any $\mathbf{x}(0) \in \mathbb{R}^{2 N}$, a small number $\delta>0$, an integer $I$, an increasing sequence of integers $\left\{k_{i}\right\}$, and a sequence of parametrization sets $\Theta(i)=\left\{\theta_{j}(i) \mid\right.$ 
TABLE VI

Mapping of Symbols From Problems $(\mathcal{P} 3)$ And $(\mathcal{P} 4)$ to Problem $(\mathcal{P} 6)$

\begin{tabular}{|c|c|c|}
\hline Problem $(\mathcal{P} 3)$ & Problem $(\mathcal{P} 6)$ & Problem $(\mathcal{P} 4)$ \\
\hline$\left[\operatorname{Re}\left\{\tilde{\mathbf{t}}_{p}^{T}\right\} \operatorname{Im}\left\{\tilde{\mathbf{t}}_{p}^{T}\right\}\right]$ & $\mathbf{x}^{T}$ & $\xi^{T}$ \\
\hline$\left\|\tilde{\mathbf{t}}_{p}\right\|_{F}^{2}$ & $\frac{1}{2} \mathbf{x}^{T} \mathbf{Q x}, \mathbf{Q}=2 \mathbf{I}$ & $\frac{1}{2} \xi^{T} \mathbf{Q} \xi, \mathbf{Q}=\operatorname{diag}(16,8,16)$ \\
\hline$-\pi, \pi$ & $\theta_{a}, \theta_{b}$ & $-\pi, \pi$ \\
\hline $\mathbf{a}(\theta)$ & $\mathbf{a}(\theta)$ & $\mathbf{g}(\theta)$ \\
\hline$e^{j p \theta}$ & $b(\theta)$ & $e^{j \theta}$ \\
\hline$\tilde{\varepsilon}_{p}$ & $\varepsilon$ & $\tilde{\varepsilon}_{1}$ \\
\hline
\end{tabular}

$\left.\theta_{j}(i) \in\left[\theta_{a}, \theta_{b}\right], j=1, \ldots, k_{i}\right\}$ such that its density distance from $\left[\theta_{a}, \theta_{b}\right]$, defined by

$$
d\left(\Theta(i),\left[\theta_{a}, \theta_{b}\right]\right) \triangleq \max _{\theta \in\left[\theta_{a}, \theta_{b}\right]} \min _{1 \leq j \leq k_{i}}\left|\theta-\theta_{j}(i)\right|
$$

satisfies

$d\left(\Theta(i),\left[\theta_{a}, \theta_{b}\right]\right) \rightarrow 0 \quad$ as $\quad i \rightarrow \infty$.

Let $\mathcal{E}(0)=\emptyset$.

Set $i$, the iteration number, to $i=0$.

Step 2) Set $i=i+1$.

Find $\mathcal{G}(i)=\{\theta \in \Theta(i) \mid \mathbf{A}(\theta) \mathbf{x}(i-1)-\mathbf{b}(\theta) \geq$ $0\} \cup \mathcal{E}(i-1)$.

Suppose $\mathcal{G}(i)$ has $l_{i}$ elements, $\mathcal{G}(i)=$ $\left\{\tilde{\theta}_{1}(i), \tilde{\theta}_{2}(i), \ldots, \tilde{\theta}_{l_{i}}(i)\right\}$.

Define $\boldsymbol{\theta}^{\left(l_{i}\right)}=\left[\begin{array}{llll}\tilde{\theta}_{1}(i) & \tilde{\theta}_{2}(i) & \ldots & \tilde{\theta}_{l_{i}}(i)\end{array}\right]^{T}$.

Step 3) Solve problem $\left(\mathcal{P} 8 l_{i}\right)$ with $\boldsymbol{\theta}^{\left(l_{i}\right)}=$ $\left[\begin{array}{llll}\tilde{\theta}_{1}(i) & \tilde{\theta}_{2}(i) & \cdots & \tilde{\theta}_{l_{i}}(i)\end{array}\right]^{T}$ to obtain an optimal solution $\left(\mathbf{x}(i), \boldsymbol{\lambda}^{\left(l_{i}\right)}\right)$.

Step 4) If $i \leq I$ or $\mid \mathbf{x}^{T}(i) \mathbf{Q x}(i)-\mathbf{x}^{T}(i-1) \mathbf{Q x}(i-$ $1) \mid \geq \delta$, find $\mathcal{E}(i)=\{\theta \in \mathcal{G}(i) \mid \mathbf{A}(\theta) \mathbf{x}(i)-$ $\mathbf{b}(\theta)=\mathbf{0}\}$ and go to step 2). Else

Step 5) Solve problem $\left(\mathcal{P} 7 l_{i}\right)$ starting from $\left(\mathbf{x}(i), \boldsymbol{\lambda}^{\left(l_{i}\right)}, \boldsymbol{\theta}^{\left(l_{i}\right)}\right)$. Denote the solution by $\left(\mathbf{x}^{*}, \boldsymbol{\lambda}^{*}, \boldsymbol{\theta}^{*}\right)$. Take $\mathbf{x}^{*}$ to be the solution of problem $(\mathcal{P} 6)$.

Theorem A2 [23]: Suppose condition (46) is satisfied. Then, the sequence $\{\mathbf{x}(i)\}$ obtained from the algorithm will converge to the solution of problem $(\mathcal{P} 6)$. Therefore, if $\delta$ and $I$ are suitably chosen, then $\mathbf{x}^{*}$ obtained in Step $\mathbf{5}$ ) is the optimal solution of $(\mathcal{P} 6)$.

Remarks:

1) As shown in [25], by solving a linear programming problem, the number of points in $\mathcal{E}(i)$ can be reduced to no more than $2 N+1$ if necessary. In case $\mathcal{G}(i)$ as defined in Step 2) has too many points, we add to $\mathcal{E}(i)$ only those points from $\Theta(i)$ at which $\left(\mathbf{x}(i), \lambda^{\left(l_{i}\right)}\right)$ violates the constraint most and the convergence result still holds. (See [25] for details.)

2) According to the theory of [23], $\operatorname{problem}\left(\mathcal{P} 8 l_{i}\right)$ in Step 3) of the algorithm can be solved with any starting point.
TABLE VII

INITIALIZATION OF DUAL PARAMETRIZATION METHOD.

\begin{tabular}{|c|c|}
\hline$k_{i}, i=0,1,2, \ldots$ & $2^{4+i}+1$ \\
\hline$\Theta(i)$ & $\left\{\theta_{j}(i)=\frac{2 \pi}{k_{i}-1} \cdot(j-1), j=1, \ldots, k_{i}\right\}$ \\
\hline$l_{0}$ & $k_{0}$ \\
\hline$\lambda_{j}(0), j=1, \ldots, k_{0}$ & {$\left[\begin{array}{llll}1 & 1 & 1 & 1\end{array}\right]^{T}$} \\
\hline$\delta$ & 0.00001 \\
\hline$I$ & 8 \\
\hline
\end{tabular}

However, by starting from the results of the previous iteration, i.e., $\left(\mathbf{x}(i-1), \boldsymbol{\lambda}^{\left(l_{i-1}\right)}\right)$, some savings in computational time can be obtained.

3) Likewise, in Step 1), one should set $\mathbf{x}(0)=$ $-\mathrm{Q}^{-1} \sum_{j=1}^{k_{0}} \mathbf{A}^{T}\left(\theta_{j}(0)\right) \boldsymbol{\lambda}_{j}(0)$, where $\lambda_{j}(0), j=$ $1, \ldots, k_{0}$, are arbitrarily chosen so that when problem $(\mathcal{P} 8 k)$ is first called, it starts at a feasible point.

\section{Implementation}

We detail here the implementation of the dual parametrization method to solve $(\mathcal{P} 3)$ and $(\mathcal{P} 4)$. Table VI summarizes the mapping of the symbols from $(\mathcal{P} 3)$ and $(\mathcal{P} 4)$ to $(\mathcal{P} 6)$, while Table VII summarizes the initialization of the algorithm.

\section{APPENDIX II \\ PROOF OF THEOREM 1}

Clearly, for any $p$ and $\theta$

$$
\left|\operatorname{Re}\left\{\tilde{e}_{p}(\theta)\right\}\right|=\left|\operatorname{Re}\left\{(-1)^{p} \tilde{e}_{p}^{*}(\theta)\right\}\right|
$$

and

$$
\left|\operatorname{Im}\left\{\tilde{e}_{p}(\theta)\right\}\right|=\left|\operatorname{Im}\left\{(-1)^{p} \tilde{e}_{p}^{*}(\theta)\right\}\right| .
$$

Therefore, we can rewrite the constraints of $(\mathcal{P} 3)$ as follows:

$$
\begin{aligned}
& \left|\operatorname{Re}\left\{(-1)^{p} \tilde{e}_{p}^{*}(\theta)\right\}\right| \leq \tilde{\varepsilon}_{p} \quad \text { and } \\
& \left|\operatorname{Im}\left\{(-1)^{p} \tilde{e}_{p}^{*}(\theta)\right\}\right| \leq \tilde{\varepsilon}_{p}, \quad \forall \theta \in[-\pi, \pi] .
\end{aligned}
$$


Next, we note $(\mathcal{P} 3)$ requires its constraints to hold for $\theta \in$ $[-\pi, \pi]$, i.e., over all azimuth angles. Accordingly, we can write the constraints of $(\mathcal{P} 3)$ equivalently as follows:

$$
\begin{aligned}
& \left|\operatorname{Re}\left\{(-1)^{p} \tilde{e}_{p}^{*}(\theta-\pi)\right\}\right| \leq \tilde{\varepsilon}_{p} \quad \text { and } \\
& \left|\operatorname{Im}\left\{(-1)^{p} \tilde{e}_{p}^{*}(\theta-\pi)\right\}\right| \leq \tilde{\varepsilon}_{p}, \quad \forall \theta \in[-\pi, \pi] .
\end{aligned}
$$

Now, from (19)

$$
\begin{aligned}
& (-1)^{p} \tilde{e}_{p}^{*}(\theta-\pi) \\
& =(-1)^{p}\left\{\sum_{n=1}^{N}\left[\tilde{\mathbf{t}}_{p}\right]_{n}^{*} \exp \left\{-j k r \cos \left(\theta-\gamma_{n}-\pi\right)\right\}-e^{-j p(\theta-\pi)}\right\} \\
& =\sum_{n=1}^{N}\left\{(-1)^{p}\left[\tilde{\mathbf{t}}_{p}\right]_{n}^{*}\right\} \exp \left\{j k r \cos \left(\theta-\gamma_{n}\right)\right\}-e^{-j p \theta}
\end{aligned}
$$

Define

$$
\mathbf{t}_{p}^{\prime}=(-1)^{p} \tilde{\mathbf{t}}_{p}^{*}
$$

Clearly

$$
\left\|\mathbf{t}_{p}^{\prime}\right\|_{F}^{2}=\left\|(-1)^{p} \tilde{\mathbf{t}}_{p}^{*}\right\|_{F}^{2}=\left\|\tilde{\mathbf{t}}_{p}\right\|_{F}^{2}
$$

It thus follows that one can write the $p$ th subproblem of $(\mathcal{P} 3)$ as follows.

\section{Problem $(\mathcal{P} 9)$ :}

$$
\min _{\mathbf{t}_{p}^{\prime}}\left\|\mathbf{t}_{p}^{\prime}\right\|_{F}^{2}
$$

subject to

$$
\left|\operatorname{Re}\left\{\sum_{n=1}^{N}\left[\mathbf{t}_{p}^{\prime}\right]_{n} \exp \left\{j k r \cos \left(\theta-\gamma_{n}\right)\right\}-e^{-j p \theta}\right\}\right| \leq \tilde{\varepsilon}_{p}
$$

and

$$
\begin{array}{r}
\left|\operatorname{Im}\left\{\sum_{n=1}^{N}\left[\mathbf{t}_{p}^{\prime}\right]_{n} \exp \left\{j k r \cos \left(\theta-\gamma_{n}\right)\right\}-e^{-j p \theta}\right\}\right| \leq \tilde{\varepsilon}_{p}, \\
\forall \theta \in[-\pi, \pi] .
\end{array}
$$

However, the $(-p)$ th subproblem of $(\mathcal{P} 3)$ is given by the following.

\section{Problem $(\mathcal{P} 10)$ :}

$$
\min _{\tilde{\mathbf{t}}_{-p}}\left\|\tilde{\mathbf{t}}_{-p}\right\|_{F}^{2}
$$

subject to

$$
\left|\operatorname{Re}\left\{\sum_{n=1}^{N}\left[\tilde{\mathbf{t}}_{-p}\right]_{n} \exp \left\{j k r \cos \left(\theta-\gamma_{n}\right)\right\}-e^{-j p \theta}\right\}\right| \leq \tilde{\varepsilon}_{-p}
$$

and

$$
\left|\operatorname{Im}\left\{\sum_{n=1}^{N}\left[\tilde{\mathbf{t}}_{-p}\right]_{n} \exp \left\{j k r \cos \left(\theta-\gamma_{n}\right)\right\}-e^{-j p \theta}\right\}\right| \leq \tilde{\varepsilon}_{-p},
$$$$
\forall \theta \in[-\pi, \pi] \text {. }
$$

Comparing $(\mathcal{P} 9)$ with $(\mathcal{P} 10)$, one sees if $\tilde{\varepsilon}_{-p}=\tilde{\varepsilon}_{p}$; then, the two subproblems are identical leading to the conclusion that $\tilde{\mathbf{t}}_{-p}=\mathbf{t}_{p}^{\prime}=(-1)^{p} \tilde{\mathbf{t}}_{p}^{*}$.
APPENDIX III

\section{PROOF OF THEOREM 2}

We begin with the following two lemmas that can be easily proved from the array geometry.

Lemma 1: The array elements of an $N$-element UCA oriented with one element at $\theta=0^{\circ}$ are distributed symmetrically about a line drawn from the center of the array at an angle of $i \pi / N, i=0, \ldots,(2 N-1)$.

Lemma 2: Consider the line of symmetry at angle $i \pi / N$. The array elements are related by

$$
\gamma_{\left(n+\left\lceil\frac{i}{2}\right\rceil-1\right) \bmod N+1} \stackrel{\bmod 2 \pi}{=} \frac{2 \pi i}{N}-\gamma_{\left(1+\left\lfloor\frac{i}{2}\right\rfloor-n\right) \bmod N+1}
$$

where $\gamma_{n}$ is the angular position of the $n$th array element as given by (2), and " $A=\bmod 2 \pi B$ " means " $A \bmod 2 \pi=B \bmod$ $2 \pi$ ".

We next prove the following result.

Theorem $C 1$ : The constraints of $(\mathcal{P} 3)$ can be written equivalently as follows:

$$
\begin{aligned}
& \left|\operatorname{Re}\left\{e^{-j q \frac{\pi}{2}} \tilde{e}_{p}^{*}\left(-\theta-\theta_{o}\right)\right\}\right| \leq \tilde{\varepsilon}_{p} \quad \text { and } \\
& \left|\operatorname{Im}\left\{e^{-j q \frac{\pi}{2}} \tilde{e}_{p}^{*}\left(-\theta-\theta_{o}\right)\right\}\right| \leq \tilde{\varepsilon}_{p}, \quad \forall \theta \in[-\pi, \pi]
\end{aligned}
$$

where $q$ is any integer and $\theta_{o}$ is any angle.

Proof: Recall the constraints of $(\mathcal{P} 3)$, which we repeat as follows:

$$
\begin{aligned}
& \left|\operatorname{Re}\left\{\tilde{e}_{p}(\theta)\right\}\right| \leq \tilde{\varepsilon}_{p} \quad \text { and } \\
& \left|\operatorname{Im}\left\{\tilde{e}_{p}(\theta)\right\}\right| \leq \tilde{\varepsilon}_{p}, \quad \forall \theta \in[-\pi, \pi] .
\end{aligned}
$$

In Appendix II, we have already seen that (58) can be written equivalently as follows (see (49)):

$$
\begin{aligned}
& \left|\operatorname{Re}\left\{( \pm 1) \cdot \tilde{e}_{p}^{*}(\theta)\right\}\right| \leq \tilde{\varepsilon}_{p} \quad \text { and } \\
& \left|\operatorname{Im}\left\{( \pm 1) \cdot \tilde{e}_{p}^{*}(\theta)\right\}\right| \leq \tilde{\varepsilon}_{p}, \quad \forall \theta \in[-\pi, \pi] .
\end{aligned}
$$

Next, observe that

$$
\begin{aligned}
& \left|\operatorname{Re}\left\{( \pm j) \cdot \tilde{e}_{p}^{*}(\theta)\right\}\right|=\left|\operatorname{Im}\left\{\tilde{e}_{p}(\theta)\right\}\right| \text { and } \\
& \left|\operatorname{Im}\left\{( \pm j) \cdot \tilde{e}_{p}^{*}(\theta)\right\}\right|=\left|\operatorname{Re}\left\{\tilde{e}_{p}(\theta)\right\}\right| .
\end{aligned}
$$

Therefore, the constraints (58) can also be written equivalently as follows:

$$
\begin{aligned}
\left|\operatorname{Im}\left\{( \pm j) \cdot \tilde{e}_{p}^{*}(\theta)\right\}\right| \leq \tilde{\varepsilon}_{p} \quad \text { and } \\
\left|\operatorname{Re}\left\{( \pm j) \cdot \tilde{e}_{p}^{*}(\theta)\right\}\right| \leq \tilde{\varepsilon}_{p}, \quad \forall \theta \in[-\pi, \pi] \\
\text { or }\left|\operatorname{Re}\left\{( \pm j) \cdot \tilde{e}_{p}^{*}(\theta)\right\}\right| \leq \tilde{\varepsilon}_{p} \quad \text { and } \\
\left|\operatorname{Im}\left\{( \pm j) \cdot \tilde{e}_{p}^{*}(\theta)\right\}\right| \leq \tilde{\varepsilon}_{p}, \quad \forall \theta \in[-\pi, \pi] .
\end{aligned}
$$

Combining (59) with (62), we obtain the following alternate expression for the constraints of $(\mathcal{P} 3)$ :

$$
\begin{aligned}
& \left|\operatorname{Re}\left\{e^{-j q \frac{\pi}{2}} \tilde{e}_{p}^{*}(\theta)\right\}\right| \leq \tilde{\varepsilon}_{p} \quad \text { and } \\
& \left|\operatorname{Im}\left\{e^{-j q \frac{\pi}{2}} \tilde{e}_{p}^{*}(\theta)\right\}\right| \leq \tilde{\varepsilon}_{p}, \quad \forall \theta \in[-\pi, \pi]
\end{aligned}
$$

where $q$ is any integer.

The result (57) follows since the constraints (63) are to hold for all angles $\theta \in[-\pi, \pi]$.

Here, we remark that the original constraints (58) and the alternative constraints (57) are fully equivalent in the sense 
that (58) implies (57), and (57) implies (58). We now prove Theorem 2.

Proof (Theorem 2): Recall the expression for $\tilde{e}_{p}(\theta)$ which we repeat below.

$$
\tilde{e}_{p}(\theta)=\sum_{n=1}^{N}\left[\tilde{\mathbf{t}}_{p}\right]_{n} \exp \left\{j k r \cos \left(\theta-\gamma_{n}\right)\right\}-e^{j p \theta} .
$$

The sum in (64) is formed by adding into it terms involving $\gamma_{1}$, then $\gamma_{2}$, then $\gamma_{3}$, etc. where $\gamma_{n}$ is the angular position of the $n$th array element. That is, we go round the UCA in an anti-clockwise direction starting from array element 1 . Clearly, we can form the same sum by going round the UCA in the clockwise direction. Moreover, we can start from any element.

Consider now the array line of symmetry at angle $i \pi / N, i \in$ $\{0, \ldots,(2 N-1)\}$ (see Lemma 1). We form the sum in (64) by going round the UCA in the anti-clockwise direction starting from the array element whose angular position is at $i \pi / N$, or just greater than $i \pi / N$ if there is no array element at $i \pi / N$. This yields

$$
\begin{aligned}
& \tilde{e}_{p}(\theta)=\sum_{n=1}^{N}\left[\tilde{\mathbf{t}}_{p}\right]_{\left(n+\left\lceil\frac{i}{2}\right\rceil-1\right) \bmod N+1} \\
& \quad \times \exp \left\{j k r \cos \left(\theta-\gamma_{\left(n+\left\lceil\frac{i}{2}\right\rceil-1\right) \bmod N+1}\right)\right\}-e^{j p \theta} .
\end{aligned}
$$

Likewise, we form the sum by going round the UCA in the clockwise direction starting from the array element whose angular position is at $i \pi / N$, or just less than $i \pi / N$ if there is no array element at $i \pi / N$. This yields

$$
\begin{aligned}
& \tilde{e}_{p}(\theta)=\sum_{n=1}^{N}\left[\tilde{\mathbf{t}}_{p}\right]_{\left(1+\left\lfloor\frac{i}{2}\right\rfloor-n\right) \bmod N+1} \\
& \quad \times \exp \left\{j k r \cos \left(\theta-\gamma_{\left.\left(1+\left\lfloor\frac{i}{2}\right\rfloor-n\right) \bmod N+1\right)}\right)\right\}-e^{j p \theta} .
\end{aligned}
$$

Substituting (56) into the exponential term of (65), we get

$$
\begin{aligned}
\tilde{e}_{p}(\theta)= & \sum_{n=1}^{N}\left[\tilde{\mathbf{t}}_{p}\right]_{\left(n+\left\lceil\frac{i}{2}\right\rceil-1\right) \bmod N+1} \\
& \times \exp \left\{j k r \cos \left(\theta-\frac{2 \pi i}{N}+\gamma_{\left.\left(1+\left\lfloor\frac{i}{2}\right\rfloor-n\right) \bmod N+1\right)}\right)\right\} \\
& -e^{j p \theta}
\end{aligned}
$$

whereupon

$$
\begin{aligned}
\tilde{e}_{p}^{*} & \left(-\theta-\pi+\frac{2 \pi i}{N}\right) \\
= & \sum_{n=1}^{N}\left[\tilde{\mathbf{t}}_{p}\right]_{\left(n+\left\lceil\frac{i}{2}\right\rceil-1\right) \bmod N+1}^{*} \\
& \times \exp \left\{j k r \cos \left(\theta-\gamma_{\left(1+\left\lfloor\frac{i}{2}\right\rfloor-n\right) \bmod N+1}\right)\right\} \\
& -e^{j p \theta} e^{j p\left(1-\frac{2 i}{N}\right) \pi}
\end{aligned}
$$

$$
\begin{aligned}
& e^{-j 2 p\left(1-\frac{2 i}{N}\right) \frac{\pi}{2}} \tilde{e}_{p}^{*}\left(-\theta-\pi+\frac{2 \pi i}{N}\right) \\
& =\sum_{n=1}^{N} e^{-j 2 p\left(1-\frac{2 i}{N}\right) \frac{\pi}{2}\left[\tilde{\mathbf{t}}_{p}\right]_{\left(n+\left\lceil\frac{i}{2}\right\rceil-1\right) \bmod N+1}^{*}} \\
& \quad \times \exp \left\{j k r \cos \left(\theta-\gamma_{\left(1+\left\lfloor\frac{i}{2}\right\rfloor-n\right) \bmod N+1}\right)\right\}-e^{j p \theta}
\end{aligned}
$$

Comparing (69) with (66), and with reference to Theorem C1, we see that we have proved (21) if $2 p\left(1-\frac{2 i}{N}\right)$ is an integer.

To complete the proof, we next show that we need only consider the lines of symmetry at angles $i \pi / N, i=0, \ldots,(N-1)$, since the symmetry relations (21) for $i=N, \ldots,(2 N-1)$ are identical to those for $i=0, \ldots,(N-1)$.

Consider the line of symmetry at angle $i \pi / N$. Its diagonally opposite line of symmetry is at angle $(i \pi / N)+\pi=$ $(i+N) \pi / N$. The symmetry relation for this new line of symmetry is given by

$$
\begin{aligned}
& {\left[\tilde{\mathbf{t}}_{p}\right]_{\left(1+\left\lfloor\frac{i+N}{2}\right\rfloor-n\right) \bmod N+1}} \\
& \quad=e^{-j 2 p\left(1-\frac{2(i+N)}{N}\right) \frac{\pi}{2}\left[\tilde{\mathbf{t}}_{p}\right]_{\left(n+\left\lceil\frac{i+N}{2}\right\rceil-1\right) \bmod N+1}^{*}} \\
& =e^{-j 2 p\left(1-\frac{2 i}{N}\right) \frac{\pi}{2}}\left[\tilde{\mathbf{t}}_{p}\right]_{\left(n+\left\lceil\frac{i+N}{2}\right\rceil-1\right) \bmod N+1}^{*} .
\end{aligned}
$$

Now, suppose $i$ is even and $N$ is odd such that

$$
\begin{aligned}
\left(1+\left\lfloor\frac{i+N}{2}\right\rfloor-n\right) \bmod N \\
=\left(1+\frac{i}{2}+\frac{N}{2}-\frac{1}{2}-n\right) \bmod N
\end{aligned}
$$

and

$$
\begin{aligned}
\left(n+\left\lceil\frac{i+N}{2}\right\rceil-1\right) \bmod N \\
=\left(n+\frac{i}{2}+\frac{N}{2}+\frac{1}{2}-1\right) \bmod N .
\end{aligned}
$$

Substituting $n=n^{\prime}-(N / 2)-(1 / 2)$, we get

$$
\begin{aligned}
(1 & \left.+\left\lfloor\frac{i+N}{2}\right\rfloor-n\right) \bmod N \\
& =\left(1+\frac{i}{2}+N-n^{\prime}\right) \bmod N \\
& =\left(1+\left\lfloor\frac{i}{2}\right\rfloor-n^{\prime}\right) \bmod N
\end{aligned}
$$

and

$$
\begin{aligned}
\left(n+\left\lceil\frac{i+N}{2}\right\rceil-1\right) \bmod N \\
=\left(n^{\prime}+\frac{i}{2}-1\right) \bmod N \\
=\left(n^{\prime}+\left\lceil\frac{i}{2}\right\rceil-1\right) \bmod N .
\end{aligned}
$$

The symmetry relation (70) can thus be written, for $i$ even and $N$ odd, as

$$
\begin{aligned}
{\left[\tilde{\mathbf{t}}_{p}\right]_{\left(1+\left\lfloor\frac{i}{2}\right\rfloor-n^{\prime}\right)} \bmod N+1 } & \\
= & e^{-j 2 p\left(1-\frac{2 i}{N}\right) \frac{\pi}{2}}\left[\tilde{\mathbf{t}}_{p}\right]_{\left(n^{\prime}+\left\lceil\frac{i}{2}\right\rceil-1\right) \bmod N+1}^{*}
\end{aligned}
$$


which is identical to the original symmetry relation (21). Using a similar technique, we can derive (75) for $i$ and $N$ both even, $i$ odd and $N$ even, and $i$ and $N$ both odd. This completes the proof.

\section{REFERENCES}

[1] Ü. Baysal and R. L. Moses, "On the geometry of isotropic wide-band arrays," in Proc. IEEE Int. Conf. Acoustics, Speech, Signal Processing (ICASSP), vol. 3, Orlando, FL, May 2002, pp. 3045-3048.

[2] M. Wax and J. Sheinvald, "Direction finding of coherent signals via spatial smoothing for uniform circular arrays," IEEE Trans. Antennas Propag., vol. 42, no. 5, pp. 613-620, May 1994.

[3] C. L. Dolph, "A current distribution for broadside arrays which optimizes the relationship between beam width and side-lobe level," in Proc. Inst. Radio Eng. (IRE), vol. 34, Jun. 1946, pp. 335-348.

[4] S. U. Pillai and B. H. Kwon, "Forward-backward spatial smoothing techniques for coherent signal identification," IEEE Trans. Acoust., Speech, Signal Process., vol. 37, no. 1, pp. 8-15, Jan. 1989.

[5] J. Xin and A. Sano, "Computationally efficient subspace-based method for direction-of-arrival estimation without eigendecomposition," IEEE Trans. Signal Process., vol. 52, no. 4, pp. 876-893, Apr. 2004.

[6] T. J. Shan and T. Kailath, "Adaptive beamforming for coherent signals and interference," IEEE Trans. Acoust., Speech, Signal Process., vol. 33, no. 3, pp. 527-536, Jun. 1985.

[7] T. P. Bronez, "Sector interpolation of nonuniform arrays for efficient high resolution bearing estimation," in Proc. IEEE Int. Conf. Acoustics, Speech, Signal Processing (ICASSP), vol. 5, New York, Apr. 1988, pp. 2885-2888.

[8] B. Friedlander, "The Root-MUSIC algorithm for direction finding with interpolated arrays," Signal Process., vol. 30, pp. 15-29, Jan. 1993.

[9] M. Pesavento, A. B. Gershman, and Z. Q. Luo, "Robust array interpolation using second-order cone programming," IEEE Signal Process. Lett., vol. 9, no. 1, pp. 8-11, Jan. 2002.

[10] G. J. Cook, B. K. Lau, and Y. H. Leung, "An alternative approach to interpolated array processing for uniform circular arrays," in Proc. IEEE Asia-Pacific Conf. Circuits Systems (APCCAS), vol. 1, Bali, Indonesia, Oct. 2002, pp. 411-414. (conference reconvened in Singapore, Dec. 2002).

[11] B. K. Lau, G. J. Cook, and Y. H. Leung, "An improved array interpolation approach to DOA estimation in Correlated Signal Environments," in Proc. IEEE Int. Conf. Acoustics, Speech, Signal Processing (ICASSP), vol. 2, Montreal, ON, Canada, May 2004, pp. 237-240.

[12] _ _ "Data-adaptive array interpolation for DOA estimation in correlated signal environments," Proc. IEEE Int. Conf. Acoustics, Speech, Signal Processing (ICASSP), vol. 4, pp. 945-948, Mar. 2005.

[13] D. E. N. Davies, "A transformation between the phasing techniques required for linear and circular aerial arrays," Proc. Inst. Elect. Eng., vol. 112, pp. 2041-2045, 1965.

[14] B. K. Lau and Y. H. Leung, "A Dolph-Chebyshev approach to the synthesis of array patterns for uniform circular arrays," in Proc. IEEE Int. Symp. Circuits Systems (ISCAS), vol. 1, Geneva, Switzerland, May 2000, pp. 124-127.

[15] C. P. Mathews and M. D. Zoltowski, "Eigenstructure techniques for 2-D angle estimation with uniform circular arrays," IEEE Trans. Signal Process., vol. 42, no. 9, pp. 2395-2407, Sep. 1994

[16] B. K. Lau and Y. H. Leung, "Optimum beamformers for uniform circular arrays in a correlated signal environment," in Proc. IEEE Int. Conf. Acoustics, Speech, Signal Processing (ICASSP), vol. 5, Istanbul, Turkey, Jun. 2000, pp. 3093-3096.

[17] M. Bührens, M. Pesavento, and J. F. Böhme, "A new approach to array interpolation by generation of artificial shift invariances: Interpolated ESPRIT," in Proc. IEEE Int. Conf. Acoustics, Speech, Signal Processing (ICASSP), vol. 5, Hong Kong, China, Apr. 2003, pp. 205-208

[18] R. Eklöf, "Dolph-Chebyshev Shading for non-ideal uniform linear arrays and non-ideal uniform circular arrays," M.Sc. thesis, ATRI, Curtin Univ. Technology, Australia, and Blekinge Inst. Technology, Sweden, Dec. 2000.
[19] M. A. Doron and E. Doron, "Wavefield modeling and array processing, part I-spatial sampling," IEEE Trans. Signal Process., vol. 42, no. 10, pp. 2549-2559, Oct. 1994.

[20] K. M. Reddy and V. U. Reddy, "Analysis of spatial smoothing with uniform circular arrays," IEEE Trans. Signal Process., vol. 47, no. 6, pp. 1726-1730, Jun. 1999.

[21] H. Krim and M. Viberg, "Two decades of array signal processing research: The parametric approach," IEEE Signal Process. Mag., vol. 13 no. 4, pp. 67-94, Jul. 1996.

[22] Y. Liu, K. L. Teo, and S. Ito, "Global optimization in linear-quadratic semi-infinite programming," Computing, pp. 119-132, 2001. Suppl. 15.

[23] Y. Liu and K. L. Teo, "An adaptive dual parametrization algorithm for quadratic semi-infinite programming problems," J. Global Optimization vol. 24, no. 2, pp. 205-217, Oct. 2002.

[24] P. Stoica and K. C. Sharman, "Novel eigenanalysis method for direction estimation," Proc. Inst. Elect. Eng., pt. F, vol. 137, pp. 19-26, Feb. 1990.

[25] Y. Liu, K. L. Teo, and S. Y. Wu, "A new quadratic semi-infinite programming algorithm based on dual parametrization," J. Global Optimization, vol. 29 , no. 4, pp. 401-413, Aug. 2004.

[26] A. Y. J. Chan and J. Litva, "MUSIC and maximum likelihood techniques on two-dimensional DOA estimation with uniform circular array," Proc. Inst. Elect. Eng.-Radar, Sonar Navigation, vol. 142, no. 3, pp. 105-114, 1995.

[27] B. Friedlander and A. J. Weiss, "Self-calibration for high-resolution array processing," in Advances in Spectrum Analysis and Array Processing, S. Haykin, Ed. Englewood Cliffs, NJ: Prentice-Hall, 1991, vol. 2, pp. 349-414.

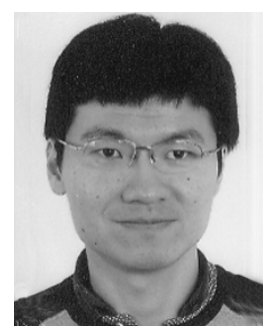

Buon Kiong Lau (M'00) received the B.E. (Hons.) degree in electrical engineering from the University of Western Australia in 1998 and the Ph.D. degree in electrical engineering from the Curtin University of Technology, Bentley, WA, Australia, in 2003.

From 2000 to 2001, he took a year off from Ph.D. studies to work as a Research Engineer at Ericsson Research, Kista, Sweden. From 2003 to 2004, he was a Guest Research Fellow at the Department of Signal Processing, Blekinge Institute of Technology, Sweden. He now holds a joint Research Fellow appointment at the Radio Systems Group and the Electromagnetic Theory Group in the Department of Electroscience, Lund University, Lund, Sweden. During 2005, he was also a Visiting Scientist at the Laboratory for Information and Decision Systems, Massachusetts Institute of Technology, Cambridge. His research interests include array signal processing, wireless communication systems, and antennas and propagation.

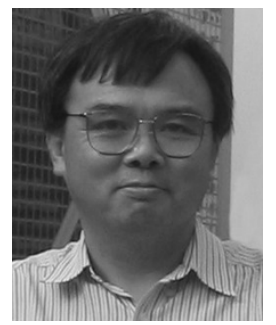

Yee Hong Leung (M'80) was born in Singapore in 1955. He received the B.E. (Hons.) and Ph.D. degrees, both in electrical engineering, from the University of Western Australia, Australia, in 1977 and 1986, respectively.

He was with the School of Electrical, Electronic and Computer Engineering, the University of Western Australia, as a Tutor from 1982 to 1986 and as a Lecturer from 1988 to 1994. From 1986 to 1988, he was a Research Scientist with the Defence Science and Technology Organization, South Australia. In 1994, he joined the Western Australian Telecommunications Research Institute, Curtin University of Technology, Perth, WA, Australia, as a Senior Research Fellow. He is also associated with the Australian Telecommunications Cooperative Research Centre. In 2004, he joined the Department of Electrical and Computer Engineering, Curtin University of Technology, as a Senior Lecturer. His research interests are in adaptive and optimum signal processing and their applications in antenna arrays and telecommunication systems. 


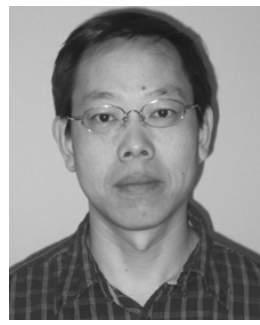

Yanqun Liu received the Ph.D. degree in mathematics from the Department of Mathematics, the University of Western Australia, Australia, in 1997.

From 1997 to 1999, he was a Research Associate with the School of Mathematics and Statistics, Curtin University of Technology, Perth, WA, Australia. From 2000 to 2003, he was a Research Fellow in the Department of Applied Mathematics and, from 2003 to 2004, a Senior Research Fellow in the Department of Electrical and Information Engineering, both at the Hong Kong Polytechnic University, Hong Kong, China. He is currently a Lecturer in the Department of Mathematics and Statistics, RMIT University, Melbourne, Australia. His research interests include optimization, optimal control, and signal processing.

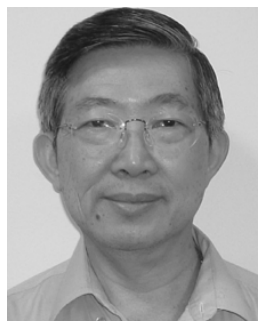

Kok Lay Teo (M'74-SM'87) was born in Johore, Malaysia, in 1946. He received the B.Sc. degree in telecommunications engineering from Ngee Ann Technical College, Singapore, in 1969 and the M.A.Sc. and Ph.D. degrees in electrical engineering from the University of Ottawa, Ottawa, ON, Canada, in 1971 and 1974, respectively.

From 1974 to 1985 , he was with the Department of Applied Mathematics, University of New South Wales, Australia, and from 1985 to 1987, he was with the Department of Industrial and Systems Engineering, National University of Singapore, Singapore. In 1988, he returned to Australia as an Associate Professor in the Department of Mathematics, University of Western Australia, Australia. In 1996, he joined the Department of Mathematics and Statistics, Curtin University of Technology, Perth, WA, Australia, as a Professor. From 1999 to 2004, he was Chair Professor of Applied Mathematics and Head of Department of Applied Mathematics at the Hong Kong Polytechnic University, China. He is currently Professor of Applied Mathematics and Head of Department of Mathematics and Statistics at Curtin University of Technology. He has delivered several keynote and fully funded invited lectures and published five books, over 250 journal papers, and a number of conference papers. The software package MISER3.3, used for solving general constrained optimal control problems, was developed by the research team under his leadership. He is Editor-in-Chief of the Journal of Industrial and Management Optimization. He also serves as an Associate Editor of a number of international journals, including Automatica; Nonlinear Dynamics and Systems Theory; Journal of Global Optimization Engineering and Optimization; Discrete and Continuous Dynamic Systems (Series A and Series B); and Dynamics of Continuous, Discrete and Impulsive Systems (Series A and Series B). He has also edited special issues for several journals, including Annals of Operations Research; Journal of Global Optimization; and Dynamics of Continuous, Discrete and Impulsive Systems (both Series A and Series B). His research interests include both the theoretical and practical aspects of optimal control and optimization and their practical applications, such as in signal processing in telecommunications and financial portfolio optimization. 